\title{
High-pressure study of the elpasolite perovskite $\mathrm{La}_{2} \mathrm{NiMnO}_{6}$
}

\author{
Christopher J. Ridley, Dominik Daisenberger, Craig Wilson, \\ Gavin B.G. Stenning, Gopinathan Sankar, Kevin S. Knight, \\ Matthew G. Tucker, Ronald I. Smith, and Craig L. Bull
}

\section{Published version information}

Citation: CJ Ridley et al. "High-pressure study of the elpasolite perovskite La2NiMnO6." Inorganic Chemistry, vol. 58, no. 14 (2019): 9016-9027.

DOI: $10.1021 /$ acs.inorgchem.9b00404

This document is the unedited author's version of a Submitted Work that was subsequently accepted for publication in Inorganic Chemistry, copyright (C2019 American Chemical Society, after peer review. To access the final edited and published work see DOI above.

Please cite only the published version using the reference above. This is the citation assigned by the publisher at the time of issuing the AAM. Please check the publisher's website for any updates. 


\title{
High-pressure study of the elpasolite perovskite $\mathrm{La}_{2} \mathrm{NiMnO}_{6}$
}

Christopher J. Ridley, ${ }^{* \dagger}$ Dominik Daisenberger, ${ }^{\ddagger}$ Craig Wilson, ${ }^{\ddagger}, \|$ Gavin B.G.

Stenning, ${ }^{\dagger}$ Gopinathan Sankar, ${ }^{\top}$ Kevin S. Knight, ${ }^{\S} \perp$ Matthew G. Tucker, ${ }^{\dagger}, \#$ Ronald I. Smith, ${ }^{\dagger}$ and Craig L. Bull ${ }^{*, \dagger}$

$\dagger$ †SIS Neutron and Muon Source, Rutherford Appleton Laboratory, Chilton, Didcot OX11 $O Q X, U K$

$\ddagger$ Diamond Light Source Ltd., Harwell Campus, Chilton, Didcot OX11 ODE, UK

IDepartment of Chemistry, University College London, 20 Gordon Street, WC1H OAJ London, U.K.

$\S$ Department of Earth Sciences, University College London, Gower Street, London, WC1E $6 B T, U K$

||Presently at: Atomic Weapons Establishment, Aldermaston, Berkshire RG7 4PR UK $\perp$ Department of Earth Sciences, The Natural History Museum, Cromwell Road, London, SW7 5BD, UK

\#Presently at: Spallation Neutron Source, One Bethel Valley Road, MS-6475, Oak Ridge, TN 37831-6475, USA.

E-mail: christopher.ridley@stfc.ac.uk; craig.bull@stfc.ac.uk

\begin{abstract}
Here we report a high-pressure investigation into the structural and magnetic properties of the double perovskite $\mathrm{La}_{2} \mathrm{NiMnO}_{6}$ using neutron scattering over a temperature
\end{abstract}


range $4.2-300 \mathrm{~K}$ at ambient pressure, and over a temperature range $120-1177 \mathrm{~K}$ up to a maximum pressure of 6.6 GPa. X-ray diffraction was also used up to a maximum pressure of $64 \mathrm{GPa}$, over a temperature range $300-720 \mathrm{~K}$. The sample was found to exist in a mixed rhombohedral/monoclinic symmetry at ambient conditions, the balance of which was found to be strongly temperature and pressure dependent. AC magnetometry, and X-ray absorption near edge structure (XANES) measurements were made at ambient pressure to characterise the sample, suggesting that the transition metal sites exist in a mixed $\mathrm{Ni}^{+3} / \mathrm{Mn}^{+3}$ and $\mathrm{Ni}^{+2} / \mathrm{Mn}^{+4}$ state at ambient temperature and pressure. Analysis of the magnetic properties of the sample shows that the Curie temperature can be enhanced by approximately $12 \mathrm{~K}$ with $2 \mathrm{GPa}$ applied pressure, but is highly stable at pressures beyond this. We report a pressure-volume-temperature equation of state for this material over this combined temperature and pressure range, with an ambient temperature bulk modulus of approximately 179(8) GPa. The previously reported transition from monoclinic to rhombohedral symmetry upon heating to $700 \mathrm{~K}$ is seen to be encouraged with applied pressure, transforming fully by approximately 1.5 GPa. Raman spectroscopy data were collected up to approximately $8 \mathrm{GPa}$ and show no clear changes or discontinuities over the reported phase transition to rhombohedral symmetry, or any indication of further changes over the range considered. The ambient pressure Grüneisen parameter, $\gamma_{t h}$, was determined to be $\gamma_{t h}=2.6$ with a Debye temperature of $677 \mathrm{~K}$. The individual modal parameters, $\gamma_{j}$ at ambient temperature were also determined from the high pressure Raman data.

\section{Introduction}

$\mathrm{La}_{2} \mathrm{NiMnO}_{6}$ has been widely studied due to its ferromagnetic-insulating nature, with a near room temperature Curie transition, ${ }^{1}$ large magnetodielectric response, ${ }^{2,3}$ magnetocaloric ef-

fect, ${ }^{4}$ and strain-induced ferroelectricity at room temperature, ${ }^{5}$ making it an interesting candidate for potential thin-film spin-based electronics. ${ }^{6,7}$ It has also attracted interest as a potential lead-free material for use in solar cells, ${ }^{8}$ and more recently for use as a cathode 
material for intermediate temperature solid-oxide fuel cells. ${ }^{9}$

Simple perovskite oxides may be described using the general formula $\mathrm{ABO}_{3}$; in the ideal undistorted case, the B-cation is 6-fold coordinated (forming octahedra), and the A-cation is 12 -fold coordinated (forming larger polyhedra) by oxygen. In a more chemically complex perovskite, there may be more than one type of cation on the B-site, which may be ordered, resulting in a doubling of the unit cell forming a double perovskite, retaining the same corner shared 6-fold octahedral structure. Materials where the mixed B-site cations don't order, show predominantly negative superexchange interactions between the transition metal (TM) cations, resulting in antiferromagnetic behaviour, ${ }^{10}$ and some component of this is seen to compete with ferromagnetic ordering with other rare-earth lanthanide double perovskites. ${ }^{11,12}$ Indeed, for $\mathrm{LaNi}_{0.5} \mathrm{Mn}_{0.5} \mathrm{O}_{3}$, the $\mathrm{Ni}-\mathrm{O}-\mathrm{Ni}$ and $\mathrm{Mn}-\mathrm{O}-\mathrm{Mn}$ bonds are believed to exhibit antiferromagnetic superexchange. ${ }^{13,14}$ However, charge ordering on the B-sites results in a positive ferromagnetic superexchange interaction (Ni-O-Mn). ${ }^{15,16}$ According to the Kanamori-Goodenough rules, ${ }^{17,18}$ ferromagnetism materialises in the case of rock-salt B-site ordering where one transition metal cation has an empty $e_{g}$ orbital, and the other has electrons in the $e_{g}$ orbital. ${ }^{11}$ This can be satisfied for $\mathrm{La}_{2} \mathrm{NiMnO}_{6}$ with either $\mathrm{Ni}^{+2} / \mathrm{Mn}^{+4}$ or $\mathrm{Ni}^{+3} / \mathrm{Mn}^{+3}$ (with $\mathrm{Mn}$ in the low spin configuration); experimentally determined literature values vary between the two configurations. ${ }^{15,19,20}$ It has been found that the exact synthesis route of the sample strongly influences the level of B-site ordering, and hence its magnetic, dielectric, and structural properties. ${ }^{8,10,21-23}$ Furthermore, the grain-size of the sample has been found to influence the magnetic properties, which has been suggested to be due to the level of antisite disorder within grains, ${ }^{24}$ with the nanoparticle form showing a highly enhanced Curie temperature. ${ }^{25}$

There have been relatively few explicit investigations into the effects of hydrostatic pressure on the crystallographic, and magnetic properties of $\mathrm{La}_{2} \mathrm{NiMnO}_{6}$. High-pressure, hightemperature synthesis has been used to control particle size to influence the magnetic properties, ${ }^{24}$ and the effects of epitaxial strain have been considered in the context of property 
enhancement for thin-films, where it was found that under tensile strain the material forms a pure monoclinic phase, whereas under compressive strain the rhombohedral symmetry is favoured. ${ }^{26}$ In addition it has been shown that epitaxially strained $\mathrm{La}_{2} \mathrm{NiMnO}_{6}$ films in the rhombohedral phase, on $\mathrm{SrTiO}_{3}$ substrates, show A-site-driven ferroelectricity, demonstrating further potential functionality in thin-films. To the best of our knowledge there has only been one study of $\mathrm{La}_{2} \mathrm{NiMnO}_{6}$ under hydrostatic pressure. Haskel et al. ${ }^{27}$ collected X-ray diffraction patterns at $200 \mathrm{~K}$ up to $50 \mathrm{GPa}$, and performed X-ray absorption nearedge structure and magnetic circular dichroism measurements up to approximately $38 \mathrm{GPa}$. The authors concluded that the ferromagnetic ground state remains stable throughout this pressure range with no discernible shift in the Curie temperature, or evidence for pressure induced valence transitions. However, only partial crystallographic data were presented on the material over these pressure ranges, with no structural information provided.

While X-rays have been used to provide an accurate measure of the volume changes in $\mathrm{La}_{2} \mathrm{NiMnO}_{6}$, they are not well suited to providing further crystallographic information on this compound, due to the similar atomic form-factors of $\mathrm{Ni}$ and $\mathrm{Mn}$, and the presence of relatively light $\mathrm{O}$ atoms along with heavier La atoms. Neutron diffraction provides much greater contrast, due to the difference in sign of the scattering length of $\mathrm{Ni}$ and $\mathrm{Mn}$, and the strong scattering from oxygen, even in the presence of heavier components of the unit cell. The aim of the present study is to investigate the nature of the structural phase boundary between the monoclinic and rhombohedral phases of $\mathrm{La}_{2} \mathrm{NiMnO}_{6}$ as a function of pressure and temperature, and to probe the correlation between this and its magnetic properties. This was achieved through a comprehensive neutron diffraction study of the material over a broad temperature and pressure range. 


\section{Synthesis and methods}

A polycrystalline sample of $\mathrm{La}_{2} \mathrm{NiMnO}_{6}$ was synthesised through the decomposition of stoichiometric mixtures of hydrated metal nitrate salts. This was achieved through first dissolving the nitrates in a minimum quantity of water in an alumina crucible with gentle heating. Once thoroughly mixed, the mixture was dried out through heating to $400 \mathrm{~K}$ over approximately $24 \mathrm{~h}$. Decomposition of the nitrates was achieved through slow heating to $900 \mathrm{~K}$ in a tube furnace in air. The samples were then annealed at $1370 \mathrm{~K}$, heated at a rate of $10 \mathrm{~K} / \mathrm{min}$, for $24 \mathrm{~h}$, before cooling at $2 \mathrm{~K} / \mathrm{min}$ back to room temperature. The resulting powder was then ground under acetone and re-annealed until no further improvement in crystallinity and phase purity was observed.

Neutron diffraction patterns were collected at the ISIS neutron and muon source; ambient pressure measurements $(4.2-300 \mathrm{~K})$ were performed on the Polaris diffractometer, and high pressure measurements were performed on the HRPD $(0-0.8 \mathrm{GPa})$ and PEARL $(0-$ $6 \mathrm{GPa}, 120-1450 \mathrm{~K}$ ) diffractometers. ${ }^{28}$ High pressure X-ray diffraction measurements were performed on the I15 beamline at the Diamond Light Source (0 - 68 GPa, $300-720$ K). Full details on the experimental methods, and high pressure techniques employed can be found in the supplementary information (SI).

\section{Results and discussion}

\section{Ambient pressure characterisation}

\section{Magnetometry}

Moment-temperature (MT) measurements at ambient pressure were performed over a range from 1.8 - $400 \mathrm{~K}$, having been both field cooled (FC), and zero field cooled (ZFC), both in applied fields of $50 \mathrm{Oe}$ and $6 \mathrm{kOe}$. These are shown in Figure 1. In low field the sample is seen to behave largely as a classical ferromagnet, showing a clear transition at approximately 
280 K. At temperatures below the Curie transition a large discrepancy between FC and ZFC moments is observed, which is removed in the presence of increased field. Moment-field (MH) measurements were performed at several temperatures to varying maximum fields between $15 \mathrm{kOe}$ and $70 \mathrm{kOe}$ (see SI).
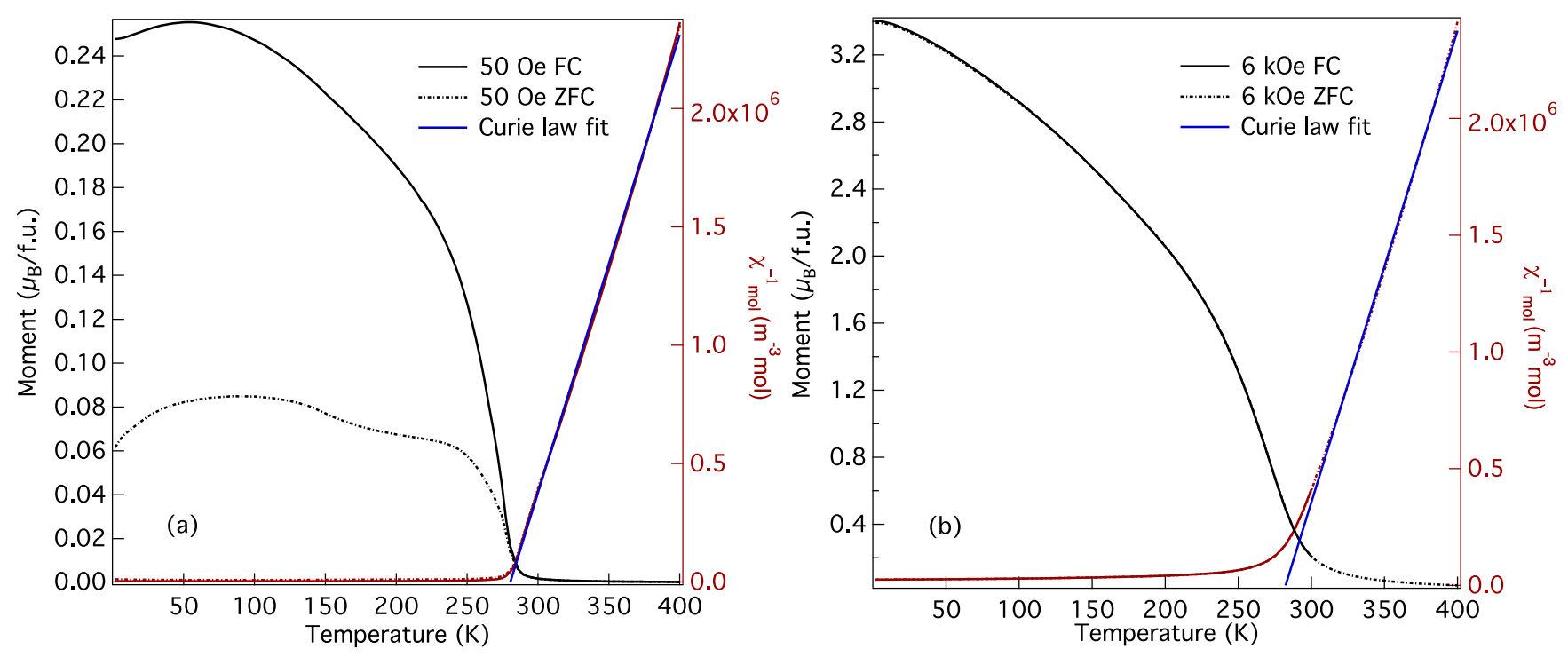

Figure 1: Moment-temperature measurements for both FC and ZFC in (a) $50 \mathrm{Oe}$ and (b) $6 \mathrm{kOe}$ applied field. The right axis in each plot shows the inverse molar susceptibility, which is fitted using the Curie law discussed in the text.

In the paramagnetic phase, the susceptibility can be fitted to the Curie law $\chi_{m}^{-1}=\frac{T-\theta}{C}$, where $\chi_{m}^{-1}$ is the inverse molar susceptibility, $\theta$ is the Curie transition temperature, and $C$ is the Curie constant, see Figure 1. In SI units, this can be related to the effective paramagnetic moment of the sample using the expression:

$$
\mu_{e f f}=\sqrt{\frac{3 k_{B} C}{\mu_{0} N_{A}}}
$$

Where $k_{B}$ is Boltzmann's constant, $N_{A}$ is Avogadro's constant, and $\mu_{0}$ is the permeability of free space. The results of these fits are summarised in Table 1, and are in reasonably good agreement with measurements from fully B-site ordered samples reported by Pal et al. ${ }^{16}$, and with other measurements in the literature. ${ }^{29}$ The determined values of the effective moment are larger than expected in the case of $\mathrm{Ni}^{2+} / \mathrm{Mn}^{4+}\left(\mu_{\text {eff }} \approx 3.4-4.7 \mu_{B}\right)$, but are closer to 
the value expected for $\mathrm{Ni}^{3+} / \mathrm{Mn}^{3+}\left(\mu_{e f f} \approx 4.4-5.8 \mu_{B}\right)$ spin states. This is consistent with earlier findings from high resolution powder diffraction ${ }^{15}$ data that there is a high degree of distortion around the $\mathrm{BO}_{6}$ octahedra indicative of a Jahn-Teller distortion due to orbital degeneracy with $\mathrm{Ni}^{3+}$ in the $t_{2 g}^{6} e_{g}^{1}$ and $\mathrm{Mn}^{3+}$ in the $t_{2 g}^{3} e_{g}^{1}$ electronic configurations respectively. For $\mathrm{Ni}^{2+}$ there are two electrons in the $e_{g}$ orbital, and there is no degeneracy to be lifted. In CGS units the Curie constant determined from the 50 Oe data is $4.136 \mathrm{emu} \mathrm{mol}^{-1} \mathrm{~K}$. This differs from the value of $3.1 \mathrm{emu} \mathrm{mol}^{-1} \mathrm{~K}$ reported by Blasse ${ }^{30}$, being more consistent with their reported theoretical value between $\mathrm{Ni}^{3+}$ (low-spin) $/ \mathrm{Mn}^{3+}$ and $\mathrm{Ni}^{3+}$ (high-spin) $/ \mathrm{Mn}^{3+}$.

The saturated magnetic moment determined from the isothermal moment-field measurement at $1.8 \mathrm{~K}$ was determined to be approximately $4.105 \mu_{\mathrm{B}} / \mathrm{fu}$. This is approximately consistent with previous reports, ${ }^{21}$ though larger than reported by Wang et al. ${ }^{22}$. This value being less than the $5 \mu_{\mathrm{B}} /$ fu predicted for spin only disordered $\mathrm{Ni}^{3+} / \mathrm{Mn}^{3+}$ is perhaps indicative of some $\mathrm{Ni}^{2+} / \mathrm{Mn}^{4+}$ character, due to the presence of antiferromagnetic antisite disorder.

Table 1: Summary of fitted and determined values from the Curie law. $\mathrm{T}_{C}$ was determined as the position of the minimum in the first derivative of the moment - temperature curve. The other parameters were fitted to the inverse susceptibility as discussed in the text. The reported uncertainties are estimated from the fits.

\begin{tabular}{ccccc}
\hline \hline Field (Oe) & $\mathrm{T}_{C}(\mathrm{~K})$ & $\theta(\mathrm{K})$ & $\mathrm{C}\left(\mathrm{m}^{3} \mathrm{~mol}^{-1} \mathrm{~K}\right)$ & $\mu_{\text {eff }}\left(\mu_{B}\right)$ \\
\hline 50 & $275(1)$ & $280.5(3)$ & $5.17(2) \times 10^{-5}$ & $5.7(3)$ \\
6000 & $275(1)$ & $282.4(4)$ & $4.95(3) \times 10^{-5}$ & $5.6(4)$ \\
\hline \hline
\end{tabular}

The moment enhancement observed in the FC data is only seen in low fields, which explains why this was not seen in the results of Rogado et al. ${ }^{1}$. AC-magnetometry was performed on the as prepared sample in order to further understand the nature of this magnetic state of the sample. The sample was measured over a frequency range from $11 \mathrm{~Hz}$ to $10 \mathrm{kHz}$, with zero DC bias field. The main features are a frequency independent peak just before the onset of the ferromagnetic transition, and a strongly frequency dependent peak at approximately $65 \mathrm{~K}$ (see Figure 2). This peak is seen to shift to higher temperature at 
higher measurement frequencies. Generally, this may be either attributed to domain wall pinning, superparamagnetism, or a spin-glass like behaviour.

The position of this peak $\left(T_{f}\right)$ was determined using two different techniques; fitting a Gaussian peak on a linear background, or through differentiating to find the turning point in the gradient of the ratio of $\mathrm{m}^{\prime \prime} / \mathrm{m}^{\prime}$ as reported in the literature. ${ }^{31}$ Both techniques yielded almost identical absolute values of $T_{f}$, but the latter resulted in more physical uncertainties, and so was used for further analysis. The Mydosh parameter, $\Phi=\frac{1}{T_{f}(\omega)} \frac{\Delta T_{f}(\omega)}{\Delta \log (\omega)}$, where $\mathrm{T}_{f}(\omega)$ is the temperature position of the peak at a given measurement frequency $\omega$, was determined to be $\Phi \approx 0.145$. This is in excellent agreement with the value reported by Yuan et al. ${ }^{31}$ $(\Phi \approx 0.13)$, and whilst $\Phi$ isn't explicitly reported by Choudhury et al. ${ }^{2}$, a value inferred from their data is consistent with the present result. This result is significantly different to the $\Phi \approx 0.02$ reported by Chandrasekhar et al. ${ }^{32}$ found from a nanoparticle study.

As previous reports had concluded that $\mathrm{La}_{2} \mathrm{NiMnO}_{6}$ shows canonical spin-glass like behaviour at low temperatures, ${ }^{32}$ the measured shift was initially fitted using the Vogel-Fulcher law,

$$
\tau=\tau_{0} \exp \left(\frac{E_{a}}{k_{B}\left(T_{f}-T_{\mathrm{vf}}\right)}\right)
$$

where $E_{a}$ is the activation energy, $\tau_{0}$ is the characteristic spin relaxation time, $T_{f}$ is the position of the frequency dependent peak, and $T_{\mathrm{vf}}$ is the Vogel-Fulcher temperature, which may be thought of as the spin-glass freezing temperature. However, the determined fit parameters converged with $T_{\mathrm{vf}} \rightarrow 0$, far lower than the typical $\mathrm{T}_{f}$ observed. This convergence leads to an Arrhenius function, which is commonly used to describe the dynamics of an assembly of non-interacting superparamagnetic particles:

$$
\tau=\tau_{0} \exp \left(\frac{E_{a}}{k_{B} T_{f}}\right)
$$

A fit to this function is shown in Figure 3. The determined fit parameters are $\tau_{0}=$ $6.411 \times 10^{-12} \mathrm{~s}, \mathrm{E}_{a} / k_{B}=1194 \mathrm{~K}\left(\mathrm{E}_{a}\right.$ equivalent to $\left.102.9 \mathrm{meV}\right)$. This indicates that the fre- 
quency dependence may originate from superparamagnetic blocking, rather than spin-glass behaviour. It should be noted that the presence of oxygen vacancies can also affect $\mathrm{AC}$ magnetisation measurements, causing local strain distortion around the vacancy which can frustrate the nearby moments. The possible concentration of defects in the present sample is discussed further on page 12 .
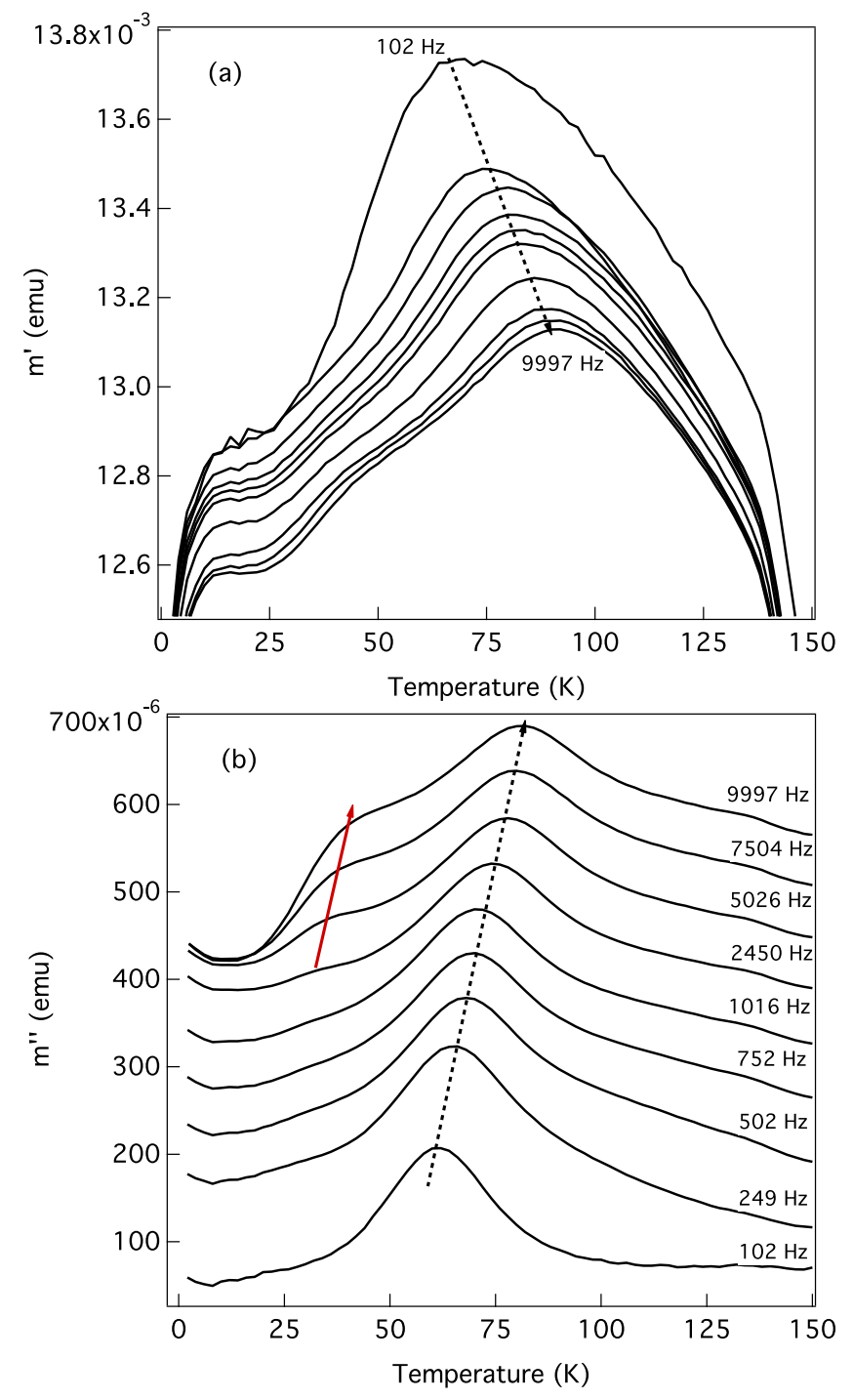

Figure 2: (a) real component and (b) imaginary component measured moment over the temperature range $2-150 \mathrm{~K}$ as measured from ACMS. In (b) the data above $102 \mathrm{~Hz}$ has been vertically offset for clarity. The dashed arrow indicates the direction of shift with increasing measurement frequency. The solid line indicates the shift of a secondary peak observable at high measurement frequencies. 


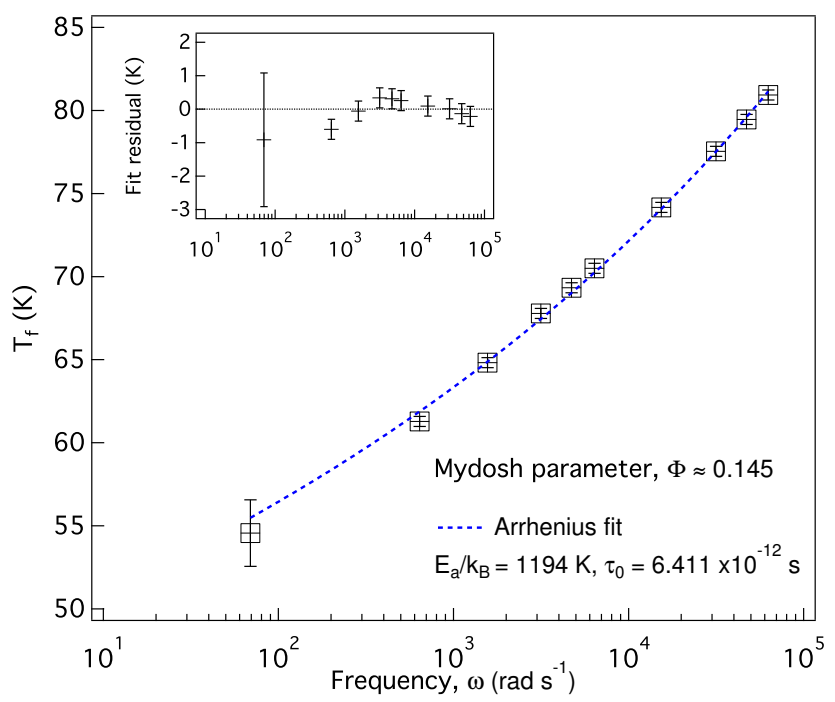

Figure 3: Fit of the Arrhenius function to the determined shift in $\mathrm{T}_{f}$. (inset) residual from fit indicating reliability of determined parameters.

The MH curves show hysteresis with a low coercive field of 250 Oe, but a large saturated moment of approximately $47 \mathrm{emu} / \mathrm{g}$ at $1.8 \mathrm{~K}$. Although it may be expected that a superparamagnet shows no hysteresis, below the blocking temperature hysteresis is expected, ${ }^{33,34}$ whilst the high saturated moment is typical. Above the blocking temperature, in this case approximately $80 \mathrm{~K}$ no hysteresis is expected. At $100 \mathrm{~K}$ the coercive field is found to be $\approx 180$ Oe, and no further change is found with increasing temperature until the sample becomes paramagnetic above the Curie transition temperature. Discrepancies between the literature values are likely caused by particle size differences due to differing material synthesis techniques.

The emergence of an additional frequency dependent response at higher measurement frequencies at approximately $40 \mathrm{~K}$ (see Figure 2 (b)), also observed by Chandrasekhar et al. ${ }^{32}$, remains unexplained. This may be due to interactions between superparamagnetic particles becoming prominent at higher frequencies, showing a form of secondary blocking temperature at lower temperatures. This theory would require additional investigation to fully explain. 


\section{XANES results}

The position and spectral profile of the L-edge provides information concerning the oxidationstate, spin-state and crystal-field of the atom being probed. The profile can be used to determine unknown oxidation states through comparison with standards of known oxidation states. Figure 4 shows the spectra as measured using the total electron yield (TEY) mode at ambient temperature and pressure, which are dominated by the $\mathrm{Ni} \mathrm{L}_{I I I}$ edge. The La $\mathrm{M}_{I V}$ edge is also expected at an energy close to $854 \mathrm{eV}$, though with a lower yield. This appears to be overlapped by the $\mathrm{Ni}_{\mathrm{L}_{I I} \text {-edge, as reported elsewhere. }}{ }^{2,35}$ The overall form of the absorption edge shows no clear change with oxidation state, however, the maximum peak position decreases in energy between +2 and +3 states. $\mathrm{LaNiO}_{2.5}$, which contains $\mathrm{Ni}$ in a mixed valence falls between the two, as expected. From this it is clear that $\mathrm{La}_{2} \mathrm{NiMnO}_{6}$ is closer in energy to a system with Ni possessing formal oxidation state of +3 , though it is suggestive that it also possesses some +2 character. This result is consistent with the findings

from the SQUID measurements, but contrasts strongly with the findings of Choudhury et al. ${ }^{2}$ for partially disordered $\mathrm{La}_{2} \mathrm{NiMnO}_{6}$, who used XAS to come to the opposite conclusion, that the sample exists in the $\mathrm{Ni}^{2+} / \mathrm{Mn}^{4+}$ state only. It is certain that the exact synthesis route has a strong influence on the electronic structure of the sample. Current findings indicate that charge ordering in the material favours the $\mathrm{Ni}^{3+} / \mathrm{Mn}^{3+}$ state. 


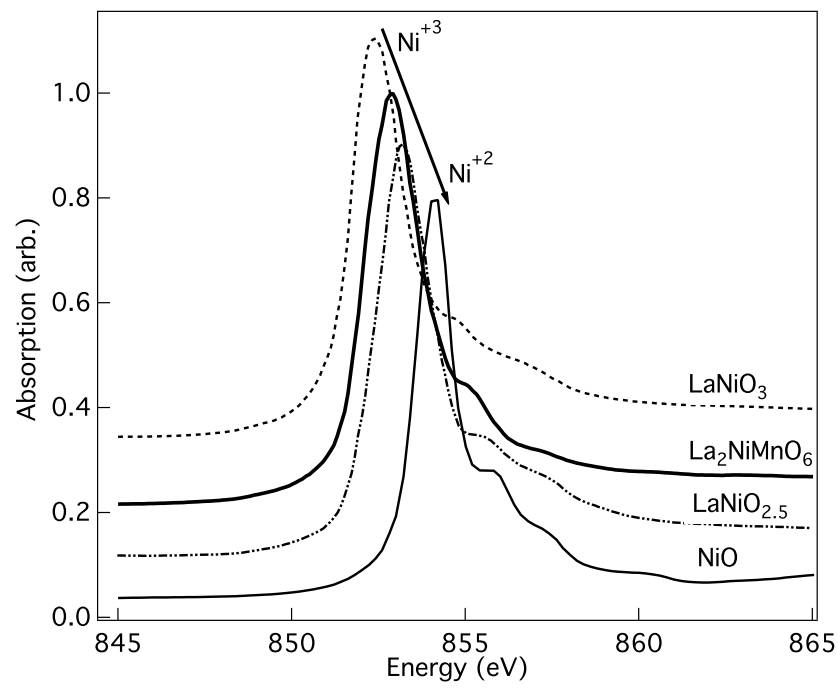

Figure 4: The Ni $\mathrm{L}_{I I}$-edge XANES spectrum of (from top to bottom) $\mathrm{LaNiO}_{3}, \mathrm{La}_{2} \mathrm{NiMnO}_{6}$, $\mathrm{LaNiO}_{2.5}$, and $\mathrm{NiO}$, at ambient pressure and temperature, showing changes in peak position upon changing oxidation state.

Pal et al. ${ }^{16}$ show similar spectra recorded in the TEY mode, and identify the $\mathrm{Ni}_{I_{I} \text {-edge }}$ at higher energy $(\approx 870 \mathrm{eV})$, but don't identify the Ni L $\mathrm{L}_{I I I}$ edge. From the present study, the spectra clearly show that the feature is present for the $\mathrm{NiO}$ standard, where there is zero contribution from La. For this reason we believe that the $\mathrm{La} \mathrm{M}_{I V}$ is present in the other spectra measured, but is weak, and overlaps with the dominant $\mathrm{Ni} \mathrm{L}_{I I I^{-}}$-edge. $\mathrm{As} \mathrm{La}^{3+}$ is an extremely stable oxidation state, no shift in energy is expected in the La $\mathrm{M}_{I V}$ edge. These spectra are therefore strongly indicative that the observed shift is entirely due to changes in the $\mathrm{Ni} \mathrm{L}_{I I}$-edge, and therefore the oxidation state of the $\mathrm{Ni}$.

\section{Low-temperature neutron diffraction}

At ambient temperature and pressure the sample is observed to be a mixture of approximately 77 wt.\% monoclinic $\left(P 2_{1} / n\right)$ and 22 wt.\% rhombohedral $(R \overline{3} r)$ symmetry with a 1 wt.\% $\mathrm{NiO}$ impurity phase. Phase coexistence in this material has been reported previously, ${ }^{21,23}$ and was attributed to inhomogeneous extrusion of the lanthanide as $\mathrm{La}_{2} \mathrm{O}_{3}$, however no additional phases are observed in the present study. The phase coexistence may therefore be a characteristic of the subtle distortive first-order nature of the transition, where 
the high temperature phase is trapped at ambient conditions even with very gradual cooling. Where there is phase coexistence with large volume difference, defects may be introduced which affect the homogeneity of the sample. In the present case, where the pseudo-cubic volume is considered (defined by $\mathrm{Ni} / \mathrm{Mn}$ sublattice), it is found to only differ by $0.3 \%$ between the two phases at $290 \mathrm{~K}$. No irregularities or discontinuities are seen in either the AC or DC magnetometry between $200-250 \mathrm{~K}$, the temperature range over which the sample is observed to become mixed phase from the monoclinic phase upon warming. This, along with a Curie temperature consistent with the literature, strongly suggests that the sample is mostly free of defects. The profile parameters of the two phases were initially constrained to be identical, and were then freely refined, yielding no improvement in fit. This indicates that the grain size of the two phases is also homogeneous.

At $4.2 \mathrm{~K}$ the sample is fully transformed into the monoclinic phase, becoming mixed phase again upon warming by $250 \mathrm{~K}$. Figure 5 shows the evolution of the diffraction pattern as a function of temperature. A clear change in intensity is seen in the $(110) /(002)$ nuclear reflections at approximately $3.87 \AA$, where the strongest magnetic contribution to the signal is seen, showing the large change in moment on the transition-metal sites. The data were fitted assuming the trivial magnetic space group $P 2_{1} / c$. No clear improvements in fit were observed with the inclusion of time reversal symmetry as for either $P 2_{1} / c^{\prime}$ or $P 2_{1}{ }^{\prime} / c$. The Ni/Mn moments were constrained to be identical between the two B-sites, with no contribution along the $a$ or $c$ axes. Subsequent relaxation of these constraints showed no improvement in the overall fit. In agreement with previous reports, ${ }^{1} \mathrm{La}_{2} \mathrm{NiMnO}_{6}$ fits with this ferromagnetic model with moments aligned along the $b$-axis. In all refinements, the $\mathrm{Ni} / \mathrm{Mn}$ occupancies were fixed at the values determined by previous high-resolution neutron powder diffraction measurements, which remains constant as a function of temperature in both phases, as reported previously. ${ }^{15}$ Full crystallographic data from these fits are provided in the SI; the evolution of the magnetic moments on each site is shown along with the change in unit cell volume in Figure 6. A change in slope of the moment versus temperature plot is seen at 
approximately $100 \mathrm{~K}$; from the magnetometry data this coincides with a region with large separation between FC and ZFC moments, and a peak in the measured response of $\chi^{\prime \prime}$. This may therefore be associated with the glass-like nature of the magnetic structure in zero field.

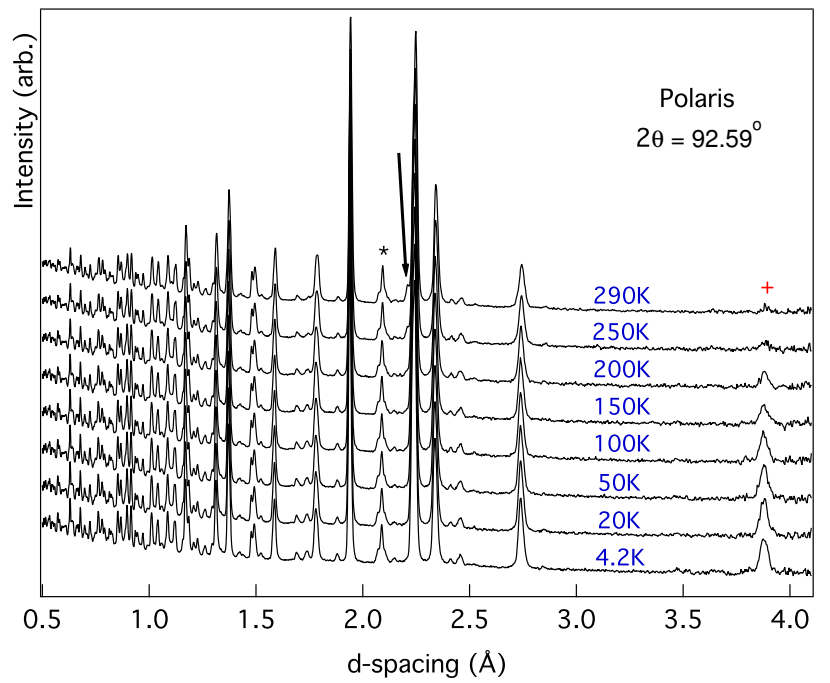

Figure 5: Temperature evolution of neutron diffraction pattern between $4.2 \mathrm{~K}$ and $300 \mathrm{~K} .(+)$ indicates strongest magnetic contribution to $(110) /(002)$ reflections. (*) indicates principal (200) reflection from NiO impurity phase. The black arrow indicates the (222) reflection of the rhombohedral phase, seen to vanish upon cooling. No magnetic signal from the $\mathrm{NiO}$ is discernible above the noise.

A simple analysis of the differential of the unit cell volume allowed for an estimate of the volumetric thermal expansion coefficient $\beta$; these values are summarised in Table 2 . The volume change as a function of temperature was fitted to an Einstein internal energy function such that $V(T)=V_{0}+\frac{\gamma_{t h}}{K_{0}} U(T)$, where $V_{0}$ is the sample volume at zero temperature, $K_{0}$ is the isothermal bulk modulus at room temperature, $\gamma_{t h}$ is the Grüneisen parameter at ambient pressure, and $U(T)$ is the internal energy function. The fit parameters were determined to be $\gamma_{t h, E}=2.4, \theta_{E}=455 \mathrm{~K}$ and $\gamma_{t h, D}=2.6, \theta_{D}=677 \mathrm{~K}$, for the Einstein and Debye approximations respectively, with $V_{T=0 \mathrm{~K}}=233.015 \AA^{3}$. The temperature evolution of the unit cell volume was analysed using $\mathrm{PASCal}^{36}$ to determine the thermal contraction along the principal axes of the system; these were found to be identical within the error of the values determined. 
Table 2: Thermal expansion coefficient determined by numerical differentiation of the data where the coefficient is calculated as, $\beta=\frac{1}{V}\left(\frac{d V}{d T}\right)$.

\begin{tabular}{ccc}
\hline \hline Temperature $(\mathrm{K})$ & $\mathrm{V}_{\text {mono }}\left(\AA^{3}\right)$ & $\beta\left(\times 10^{-5} \mathrm{~K}^{-1}\right)$ \\
\hline 290 & $234.332(5)$ & 3.858 \\
250 & $234.005(6)$ & 3.737 \\
200 & $233.545(6)$ & 3.130 \\
150 & $233.274(6)$ & 1.852 \\
100 & $233.113(6)$ & 1.030 \\
50 & $233.034(6)$ & 0.5740 \\
20 & $233.006(4)$ & 0.3092 \\
4.2 & $233.001(3)$ & 0.1358 \\
\hline \hline
\end{tabular}

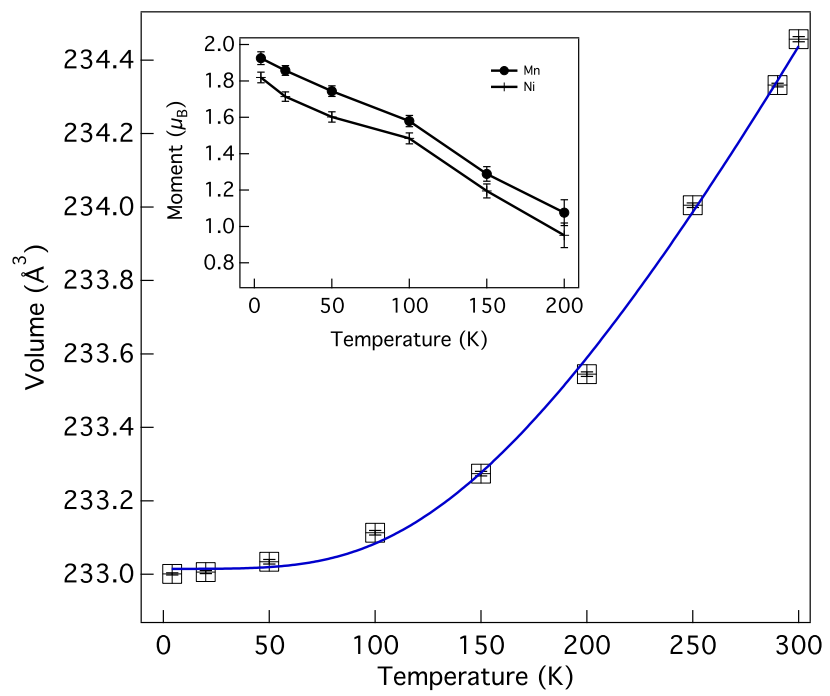

Figure 6: Change in the unit cell volume of the monoclinic phase as a function of temperature as determined from neutron diffraction. The solid line represents the fit to the Debye internal energy function as discussed in the text. (inset) evolution of refined magnetic moments on the $\mathrm{Ni} / \mathrm{Mn}$ sites.

\section{High-temperature X-ray diffraction}

Previous reports have shown that at elevated temperatures the monoclinic phase transforms to pure rhombohedral symmetry $(R \overline{3} r)$, maintaining a charge ordered state through 
the transition. ${ }^{15}$ To confirm this, high temperature X-ray diffraction measurements were performed (see Figure S1 in SI). It was observed that the sample transformed to pure rhombohedral phase by approximately $473 \mathrm{~K}$. The evolution of this phase, and the subsequent rhombohedral splitting upon further heating is shown in the SI. At the maximum temperature measured $(1373 \mathrm{~K})$ the sample remained rhombohedral. The rhombohedral angle was observed to change linearly with temperature; through extrapolation of the rhombohedral splitting to the ideal cubic value of $60^{\circ}$, it was possible to estimate that the sample should transform to expected $F m \overline{3} m$ symmetry by approximately $1950 \mathrm{~K}$. This value is consistent with findings from the literature ${ }^{23}$ and from the related perovskite $\mathrm{La}_{2} \mathrm{CoMnO}_{6}$ with the extrapolated transition temperature of approximately $1975 \mathrm{~K} .{ }^{37}$ The volumetric thermal expansion coefficient, $\beta$, of the sample in the pure rhombohedral phase was determined to be $3.421(26) \times 10^{-5} \mathrm{~K}^{-1}$, at $573 \mathrm{~K}$. This value is comparable to those reported for other elpasolite compounds, ${ }^{38,39}$ and is in reasonable agreement with the room temperature value determined from the neutron diffraction data in the mixed rhombohedral/monoclinic phase around room temperature. The mixed phase expansion coefficient may be less accurate due to the limitations in resolution.

\section{High-pressure characterisation}

\section{Magnetometry}

The shift in Curie temperature was measured as a function of pressure through determining the temperature axis intersect of the inverse susceptibility from above the transition. The general trend is shown in Figure 7 . The uncertainty on the pressures is $\pm 0.25 \mathrm{GPa}$ due to small variations in the pressure measured before and after cycling between $4 \mathrm{~K}$ and $400 \mathrm{~K}$. The uncertainty on the determined temperature is $\pm 2 \mathrm{~K}$ as determined from the linear fit to the inverse susceptibility. As the exact mass of sample in the DAC is difficult to determine, a full analysis of the magnetometry data was not possible. 


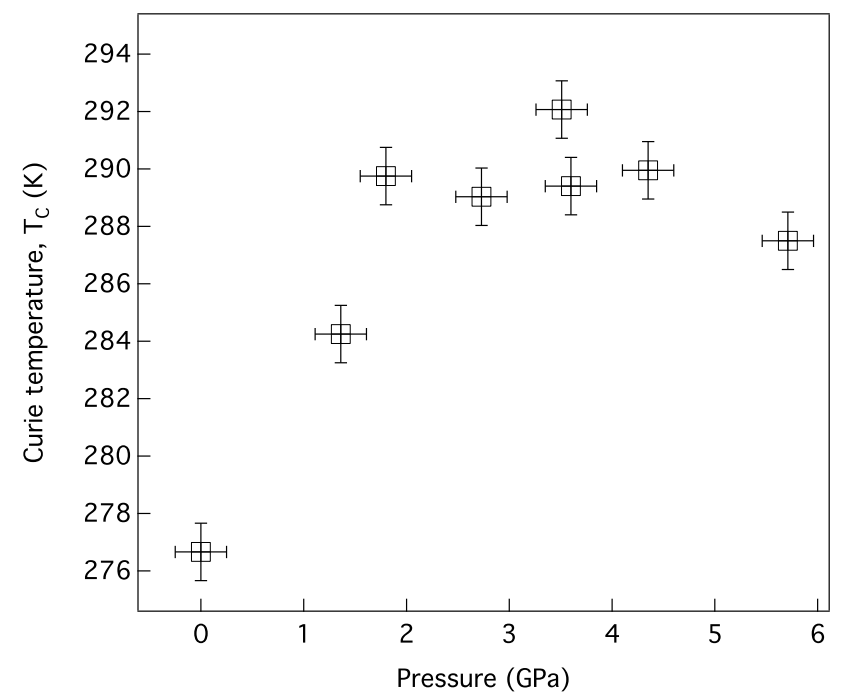

Figure 7: Variation of Curie temperature as a function of pressure. The temperature was determined from the inverse susceptibility above the transition temperature, as described in the text.

The Curie temperature is seen initially to increase with applied pressure, though above $\approx 2 \mathrm{GPa}$ there is a change in gradient, with further pressure leaving the Curie temperature effectively unchanged. This is in partial agreement with the work of Haskel et al. ${ }^{27}$, who report that the ferromagnetic ground state is unusually stable under compression to $38 \mathrm{GPa}$, from X-ray magnetic circular dichroism measurements (XMCD). However, the present study finds that under low stresses the ferromagnetic state can be altered, before entering a region of high stability. This discrepancy may be due to the relatively large errors reported from the XMCD data, or the relatively coarse pressure steps considered. ${ }^{27}$ An alternative explanation is that the level of rhombohedral/monoclinic phase coexistence is different between the two samples, and that the initial shift in Curie temperature is due to the pressure-induced phase transition between the two. This suggests that the monoclinic phase is more sensitive to pressure magnetically than the rhombohedral phase.

\section{Neutron and X-ray diffraction}

Neutron powder-diffraction gives strong sensitivity to oxygen atom positions in the presence of much heavier elements, whilst also allowing a clear distinction between $\mathrm{Ni}$ and $\mathrm{Mn}$ atomic 
positions due to large difference in their scattering lengths. Between ambient pressure and $2 \mathrm{GPa}$ the sample was observed to be a mixed phase of monoclinic $\left(P 2_{1} / n\right.$, approximately $70 \% \mathrm{wt}$ ) and rhombohedral $(R \overline{3} r)$, approximately $29 \% \mathrm{wt})$ with a $1 \%$ wt $\mathrm{NiO}$ impurity phase. Refinement of mixed phase dataset was unreliable due to the low symmetry of the primary phase, and the strong peak overlap varying with applied pressure. However, upon further compression the rhombohedral phase grows in, and by approximately $1.5 \mathrm{GPa}$ becomes the only observed phase. This is contrary to the results of density functional theory, which suggest that the monoclinic phase should be more stable at higher hydrostatic pressures. ${ }^{40}$ Enlarged regions of the profiles below this pressure around the principal reflections are shown in the SI. A LeBail fit from the present data and previous high resolution study provides insight into the changes in rhombohedral unit cell in the mixed phase region $0-0.8 \mathrm{GPa}^{41}$ (see SI), lattice parameters from the monoclinic phase were unreliable. It is seen that the rhombohedral angle increases as a function of pressure, suggesting over this pressure range the unit cell becomes more distorted, moving to lower symmetry. Full Rietveld refined data from the pure rhombohedral phase is presented in the SI. A Rydberg-Vinet equation of state with $B_{0}=179 \pm 8 \mathrm{GPa}, V_{0}=116.54 \pm 0.14 \AA^{3}$, with $B^{\prime}=4$ fixed due to a limited number of high pressure data points, was used to fit the volume reduction of the system. This is in strong agreement with the reported literature values: $B_{0}=188 \pm 28 \mathrm{GPa}, V_{0}=115.8 \pm 0.6 \AA^{3}$, with $B^{\prime}=4 \pm 1 .{ }^{27}$ The lattice parameters are plotted in Figure 8. The volume reduction above $4.5 \mathrm{GPa}$ is almost entirely taken up with reduction in the $a$ lattice parameter, with only a sub $0.1 \%$ further change in the rhombohedral angle. Interestingly, the TM-O bonds are not observed to change considerably with pressure, suggesting that the oxygen octahedra in this system are relatively rigid, with the reduction in volume taken up with the tilt of the octahedra instead. The TM-O-TM bond angle over this pressure range is seen to increase very slightly, which is consistent with the high pressure magnetometry data suggesting that there is no further change in the Curie temperature above approximately $2 \mathrm{GPa}$, as this bond mediates the superexchange between the two ferromagnetic cations. This general behaviour 
of the unit cell upon compression is reproduced from density functional theory calculations, though over a slightly larger pressure range, with an estimated peak in rhombohedral angle being reached around $10 \mathrm{GPa}$ instead of $4.5 \mathrm{GPa} .{ }^{40}$

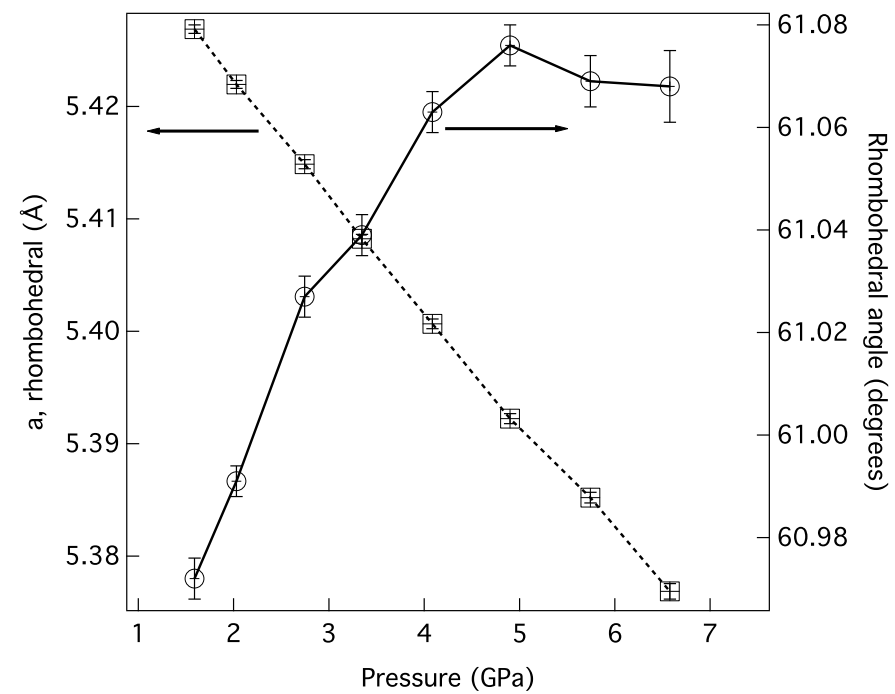

Figure 8: Rhombohedral lattice parameters shown as a function of pressure at $300 \mathrm{~K}$ as determined from neutron powder diffraction.

Over the pressure range considered for the neutron measurements, the sample wasn't seen to tend towards higher symmetry with increased compression, with the rhombohedral splitting instead increasing, and eventually remaining constant within the resolution of the diffractometer. The remaining reduction in unit cell volume is then taken with a uniform reduction in the unit cell. This is seen with regular reduction in the volume of the polyhedra. This switch into a slightly more distorted state, and the subsequent stability of this state, is likely linked to the nature of the phase transition from monoclinic to rhombohedral symmetry, and may be induced through a form of spontaneous strain in the system during this transformation. Despite this, it is partly counterintuitive that the sample isn't seen to tend towards higher symmetry with increased density, particularly as a clear linear reduction towards cubic symmetry is seen upon heating the sample at ambient pressure. To probe this further, significantly higher pressures were required, which precluded the use of neutron diffraction due to limitations in sample volume and achievable pressures. Synchrotron data 
collected at I15 was instead used. Due to the similar scattering cross sections for $\mathrm{Ni} / \mathrm{Mn}$ with X-rays, and their presence alongside relatively weakly scattering oxygen atoms, this data was limited to a LeBail extraction, providing information on the unit cell only. This data is shown in Figure 9.

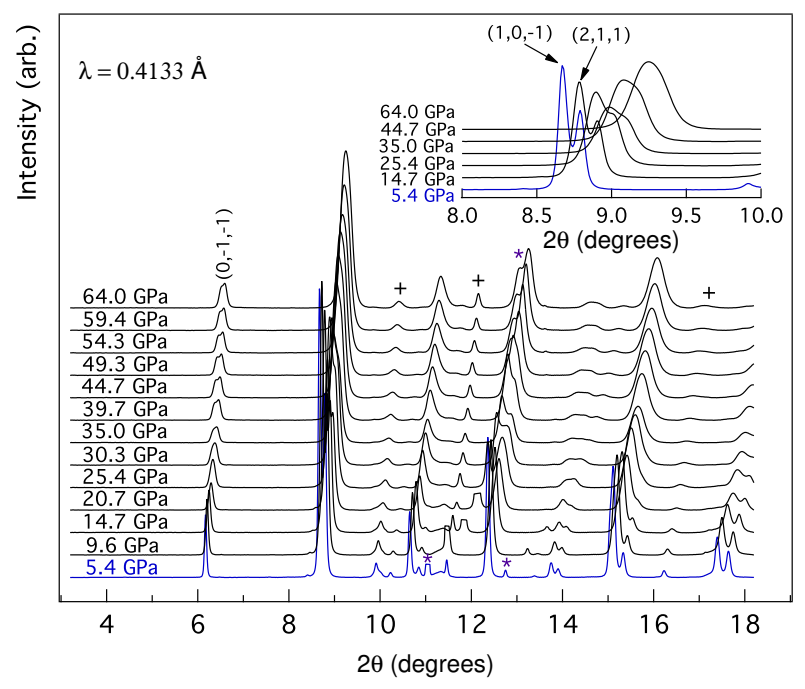

Figure 9: High pressure X-ray diffraction data collected from I15 at Diamond Light Source with $\lambda=0.4133 \AA$. Peaks indicated with $(+)$ are contributions from the $\mathrm{NiO}$ impurity phase, those indicated with $\left(^{*}\right)$ are contributions from the gasket, which have been cropped in some instances for clarity. There is an observed splitting of the $(0, \overline{1}, \overline{1})$ reflection, starting at approximately $30.3 \mathrm{GPa}$, but then clearly reduced by $64.0 \mathrm{GPa}$, the maximum pressure considered. (inset) Expanded region $8^{\circ} \leq 2 \theta \leq 10^{\circ}$ showing the rhombohedral splitting of the $(1,0, \overline{1})$, and $(2,1,1)$ reflections.

The observed splitting of the $(0, \overline{1}, \overline{1})$ reflection is believed to be due to the effects of non-hydrostaticity across the sample, as the Neon transmitting medium freezes at $4.8 \mathrm{GPa}$, remaining hydrostatic up to $15 \mathrm{GPa}$. The sample was illuminated through a $30 \mu \mathrm{m}$ pinhole, and so gradients over the sample volume would be seen as broadening of certain peaks, and offsets in the positions of longer d-spacing reflections.

The determined cell parameters are plotted in Figure 10. The volume is seen to reduce following a Rydberg-Vinet equation of state with $\mathrm{B}_{0}=175.4 \pm 7.2 \mathrm{GPa}, \mathrm{B}^{\prime}=6.19 \pm 0.33$, $\mathrm{V}_{0}=116.39 \pm 0.21 \AA^{3}$. This compares well the parameters determined over a lower pressure range from the neutron diffraction data. The rhombohedral angle is seen to show similar 
behaviour over the low pressure region, initially increasing, and then remaining essentially unchanged over the pressure region 20 - 50 GPa. However, upon further compression the rhombohedral angle is seen to reduce, essentially returning to the ambient pressure value by approximately $70 \mathrm{GPa}$. If it is assumed that the trend is now linear, then the sample would be expected to become cubic in symmetry by $200 \mathrm{GPa}$ though there isn't sufficient data to determine this with more accuracy. It is equally possible that the rhombohedral angle change remains non-linear, perhaps reaching cubic symmetry at much lower pressures. A small instrumental offset was seen between the two loadings, clearly seen in the offset of the rhombohedral angle. However, the volumes don't appear to show any clear offset within the error of the measurements. 

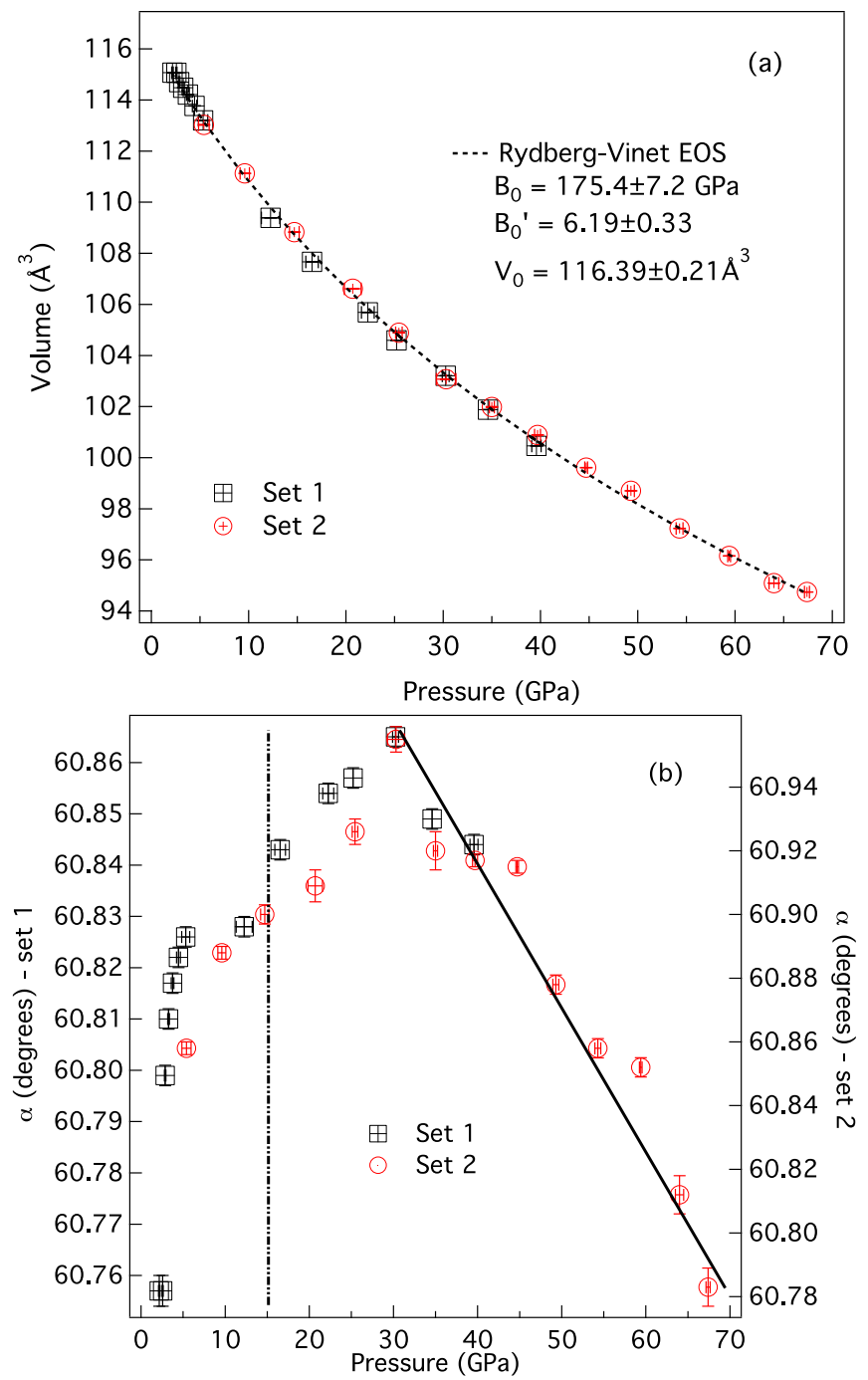

Figure 10: (a) Volume-pressure curve for the rhombohedral phase compiled from both high pressure experiments on I15. Dashed line shows a fit to the Rydberg-Vinet equation of state, with fit parameters shown. (b) Determined rhombohedral angle variation with pressure, showing eventual tendency towards the cubic aristotype. If a linear reduction in angle is assumed then extrapolation estimates cubicity by $200 \mathrm{GPa}$. The vertical dashed line indicates the approximate point at which the Ne pressure medium is only quasi-hydrostatic. The offset in the rhombohedral angle in (b) between the two loadings is discussed in the text.

As it has already been demonstrated that the phase transition from monoclinic to rhombohedral symmetry can be driven with both temperature and pressure, variable temperature measurements were performed to look for any differences in the compression mechanism. Due to the way in which the load is maintained on the Paris-Edinburgh press while the temper- 
ature is varied, there are considerable drifts in pressure upon heating or cooling. Although there is insufficient isothermal data at high pressures to perform a full comparison of the thermal expansion with that at ambient pressure, it was possible to estimate the coefficient of expansion at the highest pressure/temperature dataset considered, where the drift in pressure was within the error of the measurement. The volumetric thermal expansion, and change in rhombohedral angle $\alpha$ of the pure rhombohedral phase of the sample was determined at $1065 \mathrm{~K}$ at ambient pressure from XRD measurements, and 5.37 GPa from neutron measurements. These results are shown in Table 3.

Table 3: Comparison of the volumetric thermal expansion $(\beta)$ and the temperature dependance of the rhombohedral angle $\left(\left.\frac{d \alpha}{d T}\right|_{1065 \mathrm{~K}}\right)$ between ambient pressure (from high temperature XRD), and 5.37 GPa (from high pressure, temperature neutron diffraction).

\begin{tabular}{ccc}
\hline \hline Pressure & $\beta_{1065 \mathrm{~K}}\left(\mathrm{~K}^{-1}\right)$ & $\left.\frac{d \alpha}{d T}\right|_{1065 \mathrm{~K}}(\mathrm{deg} / \mathrm{K})$ \\
\hline Ambient & $3.397(26) \times 10^{-5}$ & $-4.8(1) \times 10^{-4}$ \\
$5.37 \mathrm{GPa}$ & $4.32(11) \times 10^{-5}$ & $-8.5(3) \times 10^{-4}$ \\
\hline \hline
\end{tabular}

The increase in thermal expansion under applied pressure is unexpected, and is perhaps due to non-hydrostatic effects, ${ }^{42}$ where the sample is under strain which is relieved with applied heat, which may be misinterpreted as additional thermal expansion. Alternatively, as one might expect much of this strain to already be relieved by $1065 \mathrm{~K}$, it is possibly due to the rhombohedral distortion of the sample at elevated pressures. The rate in reduction of $\alpha$ is seen to increase at applied pressure too, suggesting that the increased thermal expansion may be due to the sample relieving rhombohedral strain towards cubicity, from a more highly strained state.

The compiled variable temperature/pressure diffraction data was used to fit a modified Rydberg-Vinet equation of state. For this the temperature dependence of the ambient pressure volume was fitted to a second order polynomial: $V_{(P=0, T)}=V_{(P=0, T=0)}+a T+b T^{2}$ while the temperature dependence of the bulk modulus was assumed to be linear: $B=c T+d$ Due to the lack of very high pressure high temperature data the pressure derivative of the 
bulk modulus was fixed, and assumed to have no temperature dependence. The resultant fit to the data is shown in Figure 11, with nominal fit parameters: $a=0.00342 \AA^{3} \mathrm{~K}^{-1}$, $b=3.1 \times 10^{-7} \AA^{3} \mathrm{~K}^{-2}, V_{(P=0, T=0)}=115.37 \AA^{3}, c=-0.04628 \mathrm{GPaK}^{-1}, d=203.47 \mathrm{GPa} . \mathrm{A}$ full Rietveld refinement of the highest temperature neutron data was not possible due to the high attenuation from the pressure cell using tungsten carbide anvils. Lattice parameters from these measurements are reported in the SI.

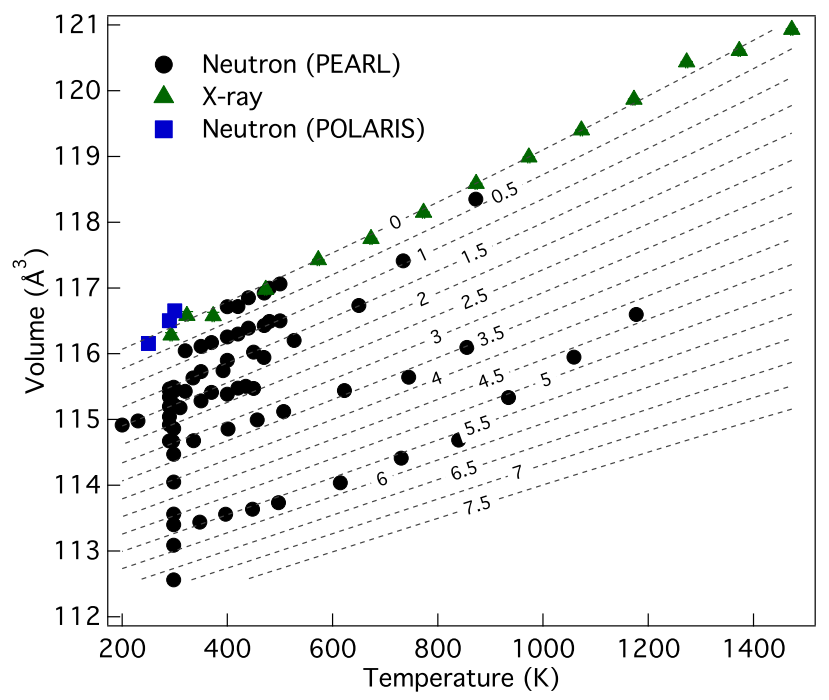

Figure 11: Compiled pressure-volume-temperature data from X-ray and neutron diffraction data from pure rhombohedral phase sample. The contours are pressures in GPa as calculated from a fit to a modified Rydberg-Vinet equation of state, as discussed in the text.

It was also possible to interpolate isothermal data points between the datasets; Figure 12 shows how the rhombohedral angle behaves under different temperature conditions. It is seen that at elevated temperatures, the rhombohedral angle becomes less sensitive to changes in pressure. This is expected, as it has already been shown that elevated temperature results in a reduced rhombohedral angle, whereas high pressure increases it. The data collected at significantly higher temperatures show a different trend, as a result of a different hydrostatic medium ( $\mathrm{MgO})$ being used, see SI. Over a much larger temperature range, it is clear that the rhombohedral angle is relatively insensitive to pressure, compared with temperature. On heating, by approximately $840 \mathrm{~K}$ the rhombohedral angle is seen to behave the same as 
under the ambient temperature conditions measured in methanol:ethanol. This is indicative of the uniaxial components of the stress being large in the sample, but being relieved under heating. Whilst full trends in the tilt angle were not possible due to the shifts in both pressure and temperature, the data suggest that at elevated temperatures, this parameter is generally seen to reduce to a less distorted state with the application of both. A provisional pressure-temperature phase diagram for the material, as determined by neutron and X-ray diffraction is shown in Figure 13.

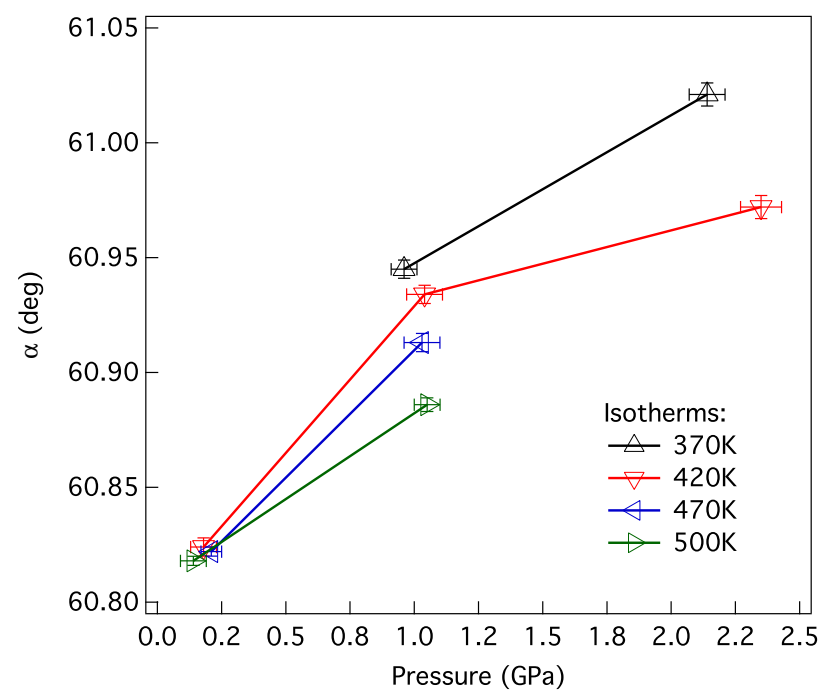

Figure 12: Isothermal behaviour of the rhombohedral angle as a function of pressure between $370-500 \mathrm{~K}$. All data were measured under highly hydrostatic conditions using methanol:ethanol as a pressure medium. 


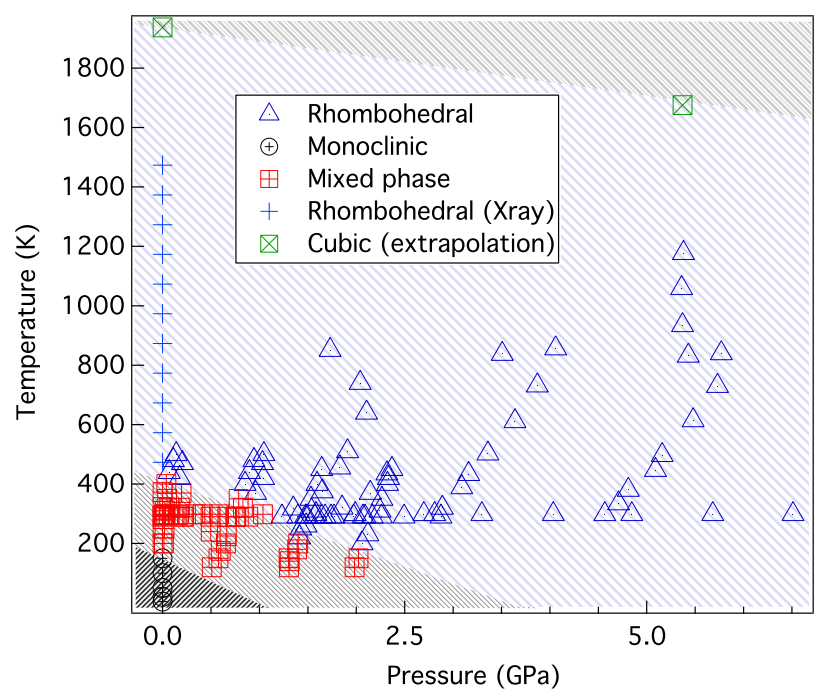

Figure 13: Structural phase diagram as determined from XRD and neutron diffraction at variable temperature and pressure. The sample is seen as phase pure monoclinic $\left(P 2_{1} / n\right)$ at low temperatures, whereas phase pure rhombohedral $(R \overline{3} r)$ symmetry is stable at elevated pressures and temperatures. There is a large mixed phase region, existing from room temperature to approximately $400 \mathrm{~K}$ at ambient pressure, and to approximately $1.5 \mathrm{GPa}$ at ambient temperature.

\section{Raman spectroscopy}

Raman spectra of $\mathrm{La}_{2} \mathrm{NiMnO}_{6}$ were collected at various pressures up to approximately $8 \mathrm{GPa}$ at ambient temperature in the range $100-800 \mathrm{rel} \mathrm{cm}^{-1}$ from the laser line $(532 \mathrm{~nm})$. Figure 14 shows a selection of the collected spectra. 

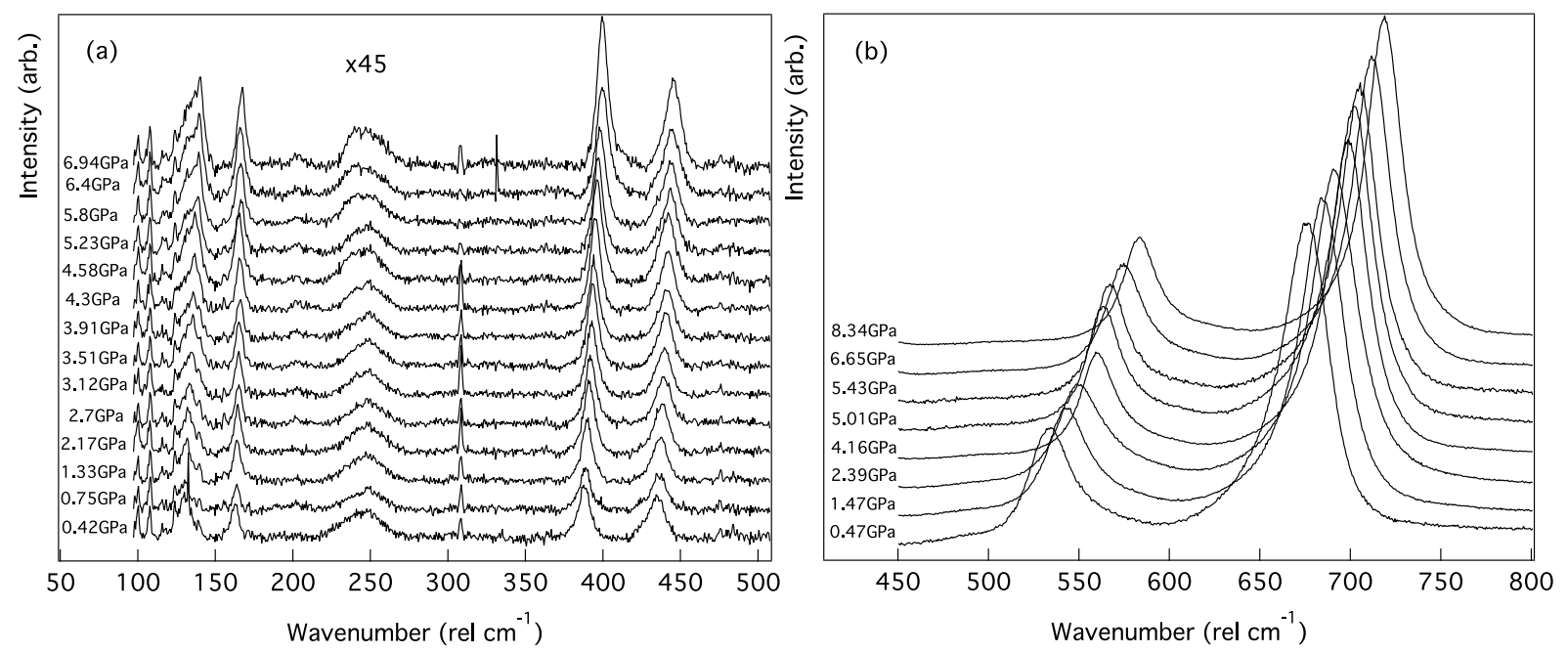

Figure 14: Compiled Raman spectra at various pressures up to approximately $8 \mathrm{GPa}$. (a) Weak peaks are found in the region $100-500 \mathrm{rel} \mathrm{cm}^{-1}$. These data have been background subtracted to clarify a comparison between them. The exposure time in this region was $15 \mathrm{~min}$. The sharp peak at approximately $310 \mathrm{rel} \mathrm{cm}^{-1}$ is an artefact from the grating in the spectrometer, which is not seen in all spectra. This peak does not shift with pressure, and is also seen when measuring the Si standard. (b) The signal is dominated by two strong peaks in the region $450-800 \mathrm{rel} \mathrm{cm}^{-1}$. The exposure time in this region was $20 \mathrm{~s}$.

The spectra are dominated by two broad and asymmetric peaks at approximately $530 \mathrm{rel} \mathrm{cm}^{-1}$ and $670 \mathrm{rel} \mathrm{cm}^{-1}$. In the lower energy region of the spectra $\left(150-500 \mathrm{rel} \mathrm{cm}^{-1}\right)$, there are a minimum of four modes which are clearly distinguishable from the noise at 433, 386, 242, and $162 \mathrm{rel} \mathrm{cm}^{-1}$. In the region below $100 \mathrm{rel} \mathrm{cm}^{-1}$ there are a cluster of additional modes which are difficult to clearly resolve. The two most clearly resolved peaks at approximately 100, $108 \mathrm{rel} \mathrm{cm}^{-1}$ are not observed to measurably shift with pressure, while the peak at approximately $128 \mathrm{rel} \mathrm{cm}^{-1}$ is observed to harden with increased pressure.

Through fitting Lorentzian profiles to the six peaks which are clearly resolved, the change in frequency of the modes was determined as a function of pressure (see SI). The pressure derivatives of each mode are summarised in Table 4. Assignment of these modes follows from the literature. ${ }^{43-46}$ The broad asymmetric peaks at higher energy were previously explained as being due to partial order on the B-site, a variable oxidation state between +2 and +4 on the B-cations, and due to different energy contributions from the Ni- and Mn-octahedra. ${ }^{45}$ Polarised Raman spectroscopy on a single crystal has provided further insight into this, 
Table 4: Summary of fitted Raman modes, their symmetry assignments following Iliev et al. ${ }^{43}$, and their pressure derivatives, and modal Grüneisen parameters, as determined from the present study.

\begin{tabular}{cccc}
\hline \hline $\begin{array}{c}\text { Position, } k \\
\text { at } 0.4 \mathrm{GPa} \\
\left.(\mathrm{rel} \mathrm{cm})^{-1}\right)\end{array}$ & Symmetry & $\begin{array}{c}d k / d P \\
\left(\mathrm{rel} \mathrm{cm} \mathrm{cm}^{-1} / \mathrm{GPa}\right)\end{array}$ & $\gamma_{j}$ \\
\hline $162.8 \pm 0.16$ & $E_{g}$ & $0.50 \pm 0.09$ & $0.71(12)$ \\
$248.3 \pm 0.5$ & $A_{g}$ & $-0.1 \pm 0.3$ & $0.1(3)$ \\
$388.1 \pm 0.1$ & $A_{g}$ & $1.7 \pm 0.1$ & $0.80(6)$ \\
$434.3 \pm 0.2$ & $A_{g}$ & $1.83 \pm 0.09$ & $0.81(4)$ \\
$534.4 \pm 0.1$ & $E_{g}$ & $6.4 \pm 0.2$ & $2.17(10)$ \\
$675.5 \pm 0.06$ & $A_{g} \& E_{g}$ & $6.4 \pm 0.2$ & $1.50(8)$ \\
\hline \hline
\end{tabular}

showing that each peak is convolved of three modes. ${ }^{44}$ This is all consistent with the fact that the structure is predominantly monoclinic $\left(P 2_{1} / n\right)$ at ambient temperature. ${ }^{15}$ In this case there was insufficient resolution to determine the shift of each of the six modes, treating each peak instead as a single convolved mode. The peak at $530 \mathrm{rel} \mathrm{cm}^{-1}$ may then be assigned to a combination of anti-stretching and bending modes, whereas the peak at $670 \mathrm{rel} \mathrm{cm}^{-1}$ is assigned to the symmetric breathing mode of the B-O octahedra. ${ }^{46,47}$

As is consistent with Grüneisen theory, the frequency of the majority of modes observed increases with reduced unit cell volume. The application of pressure to the sample has a most pronounced affect on the high energy B-O stretching modes, consistent with expectations that the sample is driven towards high symmetry with the application of pressure. Diffraction data show that the sample transitions to an almost pure rhombohedral phase between $1-1.5 \mathrm{GPa}$, no clear evidence of this is shown in the current unpolarised Raman data, due to the similarity in the energy of the modes between $P 2_{1} / n$ and $R \overline{3}$, as discussed in the literature. ${ }^{47}$ Furthermore, while the overall crystallographic symmetry of the sample is observed to change completely, it is possible the sample remains distorted locally. The individual mode Grüneisen parameters were determined as $\gamma_{j}=-\frac{d \ln \left(\nu_{j}\right)}{d \ln V}$, using the isothermal equation of state determined from the neutron diffraction data (see 48 and references 
within).

\section{Conclusion}

$\mathrm{La}_{2} \mathrm{NiMnO}_{6}$ was synthesised though a solid state nitrate decomposition route. Magnetic measurements, and XANES characterisation at ambient pressure have shown that the sample contains $\mathrm{Ni}$ in an intermediate electronic state between +3 and +2 , from which we can infer that the $\mathrm{Mn}$ is present in an electronic state between +4 and +3 . The sample was also found to have a strongly frequency dependent magnetic response, which has been fitted to an Arrhenius model. The results from these fits are consistent with either a spin-glass-like or superparamagnetic-like state. The emergence of an additional peak in the high frequency measurements suggests that inter-particle interactions may exists, such that the sample is not a perfect superparamagnet. Low-temperature neutron diffraction measurements show that the sample assumes pure monoclinic symmetry by approximately $200 \mathrm{~K}$, and a model for the ferromagnetic component of the sample has been fitted.

The previously reported temperature-driven transition from monoclinic to rhombohedral symmetry in $\mathrm{La}_{2} \mathrm{NiMnO}_{6}$ has been mapped as a function of pressure at various temperatures using both neutron and X-ray diffraction. A model for the pressure-volume-temperature equation of state of the pure rhombohedral phase material has been proposed. A large region of mixed phase is found above ambient conditions. The pure rhombohedral phase is found to be highly stable, with no clear reduction in the rhombohedral angle towards a higher symmetry until pressures beyond $50 \mathrm{GPa}$, this is found in sharp contrast to its relatively high sensitivity to temperature at ambient pressure. Under ambient temperature, the extrapolated cubic transition is expected around $200 \mathrm{GPa}$. The Curie temperature of the sample was measured as a function of pressure, and is shown to increase with pressure below 1.5 GPa, before becoming very stable under further compression. This is shown to be related to the region of phase coexistence between rhombohedral/monoclinic symmetry, and also 
related to the superexchange angle. High pressure Raman measurements were performed, and show no clear indication of the transition from monoclinic to rhombohedral symmetry on the local scale. This is due to the very subtle crystallographic distortion resulting in similar mode energies either side of the transition, within the resolution of the spectrometer.

\section{Acknowledgement}

The authors acknowledge the Science and Technology Facilities Council for providing neutron beamtime on the Polaris, HRPD, and PEARL instruments at the ISIS Neutron and Muon Source, and the I15 instrument at the Diamond Light Source under proposal number EE11828, and thank Daniel Nye for his assistance with the SQUID and X-ray diffraction measurements in the Materials Characterisation Laboratory at the ISIS Neutron and Muon Source. The authors also acknowledge Dominic Fortes, and Alex Gibbs for their assistance with the measurements on HRPD.

\section{Supporting Information Available}

Ambient pressure SQUID measurements were performed in a gelatin capsule, no diamagnetic correction for the sample straw holder was performed. High pressure SQUID momenttemperature data were background subtracted using the empty pressure cell. Further details of the sample synthesis, and full details of the experimental techniques are provided. Additional X-ray and neutron powder diffraction data are presented, with refined crystallographic information. Additional magnetometry and Raman data are also shown.

\section{References}

(1) Rogado, N. S.; Li, J.; Sleight, A. W.; Subramanian, M. A. Magnetocapacitance and magnetoresistance near room temperature in a ferromagnetic semiconductor: 
$\mathrm{La}_{2} \mathrm{NiMnO}_{6}$. Advanced Materials 2005, 17, 2225-2227.

(2) Choudhury, D.; Mandal, P.; Mathieu, R.; Hazarika, A.; Rajan, S.; Sundaresan, A.; Waghmare, U. V.; Knut, R.; Karis, O.; Nordblad, P.; Sarma, D. D. Near-roomtemperature colossal magnetodielectricity and multiglass properties in partially disordered $\mathrm{La}_{2} \mathrm{NiMnO}_{6}$. Physical Review Letters 2012, 108, 127201.

(3) Chandrasekhar, K. D.; Das, A. K.; Mitra, C.; Venimadhav, A. The extrinsic origin of the magnetodielectric effect in the double perovskite $\mathrm{La}_{2} \mathrm{NiMnO}_{6}$. Journal of Physics: Condensed Matter 2012, 24, 495901.

(4) Balli, M.; Fournier, P.; Jandl, S.; Gospodinov, M. M. A study of the phase transition and magnetocaloric effect in multiferroic $\mathrm{La}_{2} \mathrm{MnNiO}_{6}$ single crystals. Journal of Applied Physics 2014, 115, 173904.

(5) Takahashi, R.; Ohkubo, I.; Yamauchi, K.; Kitamura, M.; Sakurai, Y.; Oshima, M.; Oguchi, T.; Cho, Y.; Lippmaa, M. A-site-driven ferroelectricity in strained ferromagnetic $\mathrm{La}_{2} \mathrm{NiMnO}_{6}$ thin films. Physical Review B 2015, 91, 134107.

(6) Kitamura, M.; Ohkubo, I.; Kubota, M.; Matsumoto, Y.; Koinuma, H.; Oshima, M. Ferromagnetic properties of epitaxial $\mathrm{La}_{2} \mathrm{NiMnO}_{6}$ thin films grown by pulsed laser deposition. Applied Physics Letters 2009, 94, 132506.

(7) Guo, L.; Bai, Y.; Huang, C.; Ma, W. Revisiting $\mathrm{La}_{2} \mathrm{MMnO}_{6}(\mathrm{M}=\mathrm{Co}, \mathrm{Ni}, \mathrm{Cu}, \mathrm{Zn})$ perovskites in view of 3d-electron configuration. Journal of Applied Physics 2018, 124, 065103.

(8) Lan, C.; Zhao, S.; Xu, T.; Ma, J.; Hayase, S.; Ma, T. Investigation on structures, band gaps, and electronic structures of lead free $\mathrm{La}_{2} \mathrm{MnNiO}_{6}$ double perovskite materials for potential application of solar cell. Journal of Alloys and Compounds 2016, 655, 208-214. 
(9) Sun, L.-P.; Li, H.; Li, Q.; Huo, L.-H.; Zhao, H.; Bassat, J.-M.; Rougier, A.; Fourcade, S.; Grenier, J.-C. Evaluation of $\mathrm{La}_{2-x} \mathrm{NiMnO}_{6-\delta}$ as cathode for intermediate temperature solid oxide fuel cells. Journal of Power Sources 2018, 392, 8-14.

(10) Singh, M. P.; Truong, K. D.; Jandl, S.; Fournier, P. Long-range Ni/Mn structural order in epitaxial double perovskite $\mathrm{La}_{2} \mathrm{NiMnO}_{6}$ thin films. Physical Review B 2009, 79, 224421.

(11) Yi, W.; Liang, Q.; Matsushita, Y.; Tanaka, M.; Belik, A. A. High-pressure synthesis, crystal structure, and properties of $\mathrm{In}_{2} \mathrm{NiMnO}_{6}$ with antiferromagnetic order and fieldinduced phase transition. Inorganic Chemistry 2013, 52, 14108-14115.

(12) Retuerto, M.; Munoz, A.; Martínez-Lope, M. J.; Alonso, J. A.; Mompean, F. J.; Fernández-Díaz, M. T.; Sánchez-Benítez, J. Magnetic interactions in the double perovskites $\mathrm{R}_{2} \mathrm{NiMnO}_{6}(\mathrm{R}=\mathrm{Tb}, \mathrm{Ho}, \mathrm{Er}, \mathrm{Tm})$ investigated by neutron diffraction. Inorganic Chemistry 2015, 54, 10890-10900.

(13) Wold, A.; Arnott, R. J.; Goodenough, J. B. Some Magnetic and Crystallographic Properties of the System LaMn ${ }_{1-x} \mathrm{Ni}_{x} \mathrm{O}_{3+\lambda}$. Journal of Applied Physics 1958, 29, 387-389.

(14) Joly, V. L. J.; Joy, P. A.; Date, S. K.; Gopinath, C. S. Two ferromagnetic phases with different spin states of $\mathrm{Mn}$ and $\mathrm{Ni}$ in $\mathrm{LaMn}_{0.5} \mathrm{Ni}_{0.5} \mathrm{O}_{3}$. Physical Review B 2002, 65, 184416.

(15) Bull, C. L.; Gleeson, D.; Knight, K. S. Determination of B-site ordering and structural transformations in the mixed transition metal perovskites $\mathrm{La}_{2} \mathrm{CoMnO}_{6}$ and $\mathrm{La}_{2} \mathrm{NiMnO}_{6}$. Journal of Physics: Condensed Matter 2003, 15, 4927.

(16) Pal, S.; Govinda, S.; Goyal, M.; Mukherjee, S.; Pal, B.; Saha, R.; Sundaresan, A.; Jana, S.; Karis, O.; Freeland, J. W.; Sarma, D. D. Effect of anti-site disorder on magnetism in $\mathrm{La}_{2} \mathrm{NiMnO}_{6}$. Physical Review B 2018, 97, 165137. 
(17) Goodenough, J. B. Theory of the role of covalence in the perovskite-type manganites [La, M(II)] Mn $\mathrm{O}_{3}$. Physical Review 1955, 100, 564 .

(18) Kanamori, J. Superexchange interaction and symmetry properties of electron orbitals. Journal of Physics and Chemistry of Solids 1959, 10, 87-98.

(19) Sánchez, M.; García, J.; Blasco, J.; Subías, G.; Perez-Cacho, J. Local electronic and geometrical structure of $\mathrm{LaNi}_{1-x} \mathrm{Mn}_{x} \mathrm{O}_{3+\delta}$ perovskites determined by X-ray-absorption spectroscopy. Physical Review B 2002, 65, 144409.

(20) Gauvin-Ndiaye, C.; Baker, T. E.; Karan, P.; Massé, E.; Balli, M.; Brahiti, N.; Eskandari, M. A.; Fournier, P.; Tremblay, A.-M. S.; Nourafkan, R. Electronic and magnetic properties of the candidate magnetocaloric-material double perovskites $\mathrm{La}_{2} \mathrm{MnCoO}_{6}$, $\mathrm{La}_{2} \mathrm{MnNiO}_{6}$, and $\mathrm{La}_{2} \mathrm{MnFeO}_{6}$. Physical Review B 2018, 98, 125132.

(21) Dass, R. I.; Yan, J.-Q.; Goodenough, J. B. Oxygen stoichiometry, ferromagnetism, and transport properties of $\mathrm{La}_{2-x} \mathrm{NiMnO}_{6+\delta}$. Physical Review B 2003, 68, 064415.

(22) Wang, X.; Sui, Y.; Li, Y.; Li, L.; Zhang, X.; Wang, Y.; Liu, Z.; Su, W.; Tang, J. The influence of the antiferromagnetic boundary on the magnetic property of $\mathrm{La}_{2} \mathrm{NiMnO}_{6}$. Applied Physics Letters 2009, 95, 252502.

(23) Sayed, F. N.; Achary, S. N.; Jayakumar, O. D.; Deshpande, S. K.; Krishna, P. S. R.; Chatterjee, S.; Ayyub, P.; Tyagi, A. K. Role of annealing conditions on the ferromagnetic and dielectric properties of $\mathrm{La}_{2} \mathrm{NiMnO}_{6}$. Journal of Materials Research 2011, 26, $567-577$.

(24) Gan, H.; Wang, C. B.; Li, L.; Shen, Q.; Zhang, L. M. Structural and magnetic properties of $\mathrm{La}_{2} \mathrm{NiMnO}_{6}$ ceramic prepared by ultra-high pressure sintering. Journal of Alloys and Compounds 2018, 735, 2486-2490. 
(25) Ravi, S.; Senthilkumar, C. Room temperature ferromagnetism with high Curie temperature in $\mathrm{La}_{2} \mathrm{NiMnO}_{6}$ nanoparticle. Materials Letters 2016, 164, 124-126.

(26) Wu, S.-Q.; Cheng, S.; Lu, L.; Liu, M.; Jin, X.-W.; Cheng, S.-D.; Mi, S.-B. B-site ordering and strain-induced phase transition in double-perovskite $\mathrm{La}_{2} \mathrm{NiMnO}_{6}$ films. Scientific reports 2018, 8, 2516.

(27) Haskel, D.; Fabbris, G.; Souza-Neto, N. M.; Van Veenendaal, M.; Shen, G.; Smith, A. E.; Subramanian, M. Stability of the ferromagnetic ground state of $\mathrm{La}_{2} \mathrm{MnNiO}_{6}$ against large compressive stress. Physical Review B 2011, 84, 100403.

(28) Bull, C. L.; Funnell, N. P.; Tucker, M. G.; Hull, S.; Francis, D. J.; Marshall, W. G. PEARL: the high pressure neutron powder diffractometer at ISIS. High Pressure Research 2016, 36, 493-511.

(29) Luo, X.; Wang, B.; Sun, Y. P.; Zhu, X. B.; Song, W. H. Critical behavior of double perovskite $\mathrm{La}_{2} \mathrm{NiMnO}_{6}$. Journal of Physics: Condensed Matter 2008, 20, 465211.

(30) Blasse, G. Ferromagnetic interactions in non-metallic perovskites. Journal of physics and Chemistry of Solids 1965, 26, 1969-1971.

(31) Yuan, X.; Li, Q.; Hu, J.; Xu, M. Unusual dynamic magnetic behavior of polycrystalline $\mathrm{La}_{2} \mathrm{NiMnO}_{6}$. Physica B: Condensed Matter 2013, 424, 73-78.

(32) Chandrasekhar, K. D.; Das, A. K.; Venimadhav, A. Spin glass behaviour and extrinsic origin of magnetodielectric effect in non-multiferroic $\mathrm{La}_{2} \mathrm{NiMnO}_{6}$ nanoparticles. Journal of Physics: Condensed Matter 2012, 24, 376003.

(33) Mamiya, H.; Jeyadevan, B. Magnetic hysteresis loop in a superparamagnetic state. IEEE Transactions on Magnetics 2014, 50, 1-4.

(34) Bedanta, S.; Kleemann, W. Supermagnetism. Journal of Physics D: Applied Physics 2008, 42, 013001. 
(35) Gibert, M.; Viret, M.; Torres-Pardo, A.; Piamonteze, C.; Zubko, P.; Jaouen, N.; Tonnerre, J.-M.; Mougin, A.; Fowlie, J.; Catalano, S.; Gloter, A.; StỐphan, O.; Triscone, J.M. Interfacial Control of Magnetic Properties at LaMnO3/LaNiO3 Interfaces. Nano Letters 2015, 15, 7355-7361.

(36) Cliffe, M. J.; Goodwin, A. L. PASCal: a principal axis strain calculator for thermal expansion and compressibility determination. Journal of Applied Crystallography 2012, $45,1321-1329$.

(37) Bull, C. L.; Playford, H. Y.; Knight, K. S.; Stenning, G. B. G.; Tucker, M. G. Magnetic and structural phase diagram of the solid solution $\mathrm{LaCo}_{x} \mathrm{Mn}_{1-x} \mathrm{O}_{3}$. Physical Review B 2016, 94, 014102 .

(38) Filonova, E. A.; Skutina, L. S.; Medvedev, D. A. Phase transitions and thermal expansion of Sr2-xBaxNiMoO6 and Sr2Ni1-yZnyMoO6 solid solutions. Inorganic Materials 2016, 52, 57-62.

(39) Wei, T.; Zhang, Q.; Huang, Y.-H.; Goodenough, J. B. Cobalt-based double-perovskite symmetrical electrodes with low thermal expansion for solid oxide fuel cells. Journal of Materials Chemistry 2012, 22, 225-231.

(40) Yang, D.; Wang, W.; Yang, T.; Lampronti, G. I.; Ye, H.; Wu, L.; Yu, Q.; Lu, S. Role of spontaneous strains on the biphasic nature of partial B-site disorder double perovskite $\mathrm{La}_{2} \mathrm{NiMnO}_{6}$. APL Materials 2018, 6, 066102.

(41) Bull, C. L.; Fortes, A. D.; Ridley, C. J.; Wood, I. G.; Dobson, D. P.; Funnell, N. P.; Gibbs, A. S.; Goodway, C. M.; Sadykov, R.; Knight, K. S. High-resolution neutrondiffraction measurements to 8 kbar. High Pressure Research 2017, 37, 486-494.

(42) The sample is compressed with $\mathrm{MgO}$ as a pressure medium in the very high temperature measurements, as the gaskets cannot seal fluid media. 
(43) Iliev, M. N.; Abrashev, M. V.; Litvinchuk, A. P.; Hadjiev, V. G.; Guo, H.; Gupta, A. Raman spectroscopy of ordered double perovskite $\mathrm{La}_{2} \mathrm{CoMnO}_{6}$ thin films. Physical Review B 2007, 75, 104118.

(44) Iliev, M. N.; Gospodinov, M. M.; Singh, M. P.; Meen, J.; Truong, K. D.; Fournier, P.; Jandl, S. Growth, magnetic properties, and Raman scattering of $\mathrm{La}_{2} \mathrm{NiMnO}_{6}$ single crystals. Journal of Applied Physics 2009, 106, 023515.

(45) Bull, C. L.; McMillan, P. F. Raman scattering study and electrical properties characterization of elpasolite perovskites $\mathrm{Ln}_{2}\left(\mathrm{BB}^{\prime}\right) \mathrm{O}_{6}\left(\mathrm{Ln}=\mathrm{La}, \mathrm{Sm} . . \mathrm{Gd}\right.$ and $\mathrm{B}, \mathrm{B}^{\prime}=\mathrm{Ni}$, Co, Mn). Journal of Solid State Chemistry 2004, 177\%, 2323-2328.

(46) Martín-Carrón, L.; De Andres, A.; Martínez-Lope, M. J.; Casais, M. T.; Alonso, J. A. Raman phonons as a probe of disorder, fluctuations, and local structure in doped and undoped orthorhombic and rhombohedral manganites. Physical Review B 2002, 66, 174303.

(47) Iliev, M. N.; Guo, H.; Gupta, A. Raman spectroscopy evidence of strong spin-phonon coupling in epitaxial thin films of the double perovskite $\mathrm{La}_{2} \mathrm{NiMnO}_{6}$. Applied physics letters 2007, 90, 151914.

(48) Vocadlo, L.; Poirer, J. P.; Price, G. D. Gruneisen parameters and isothermal equations of state. American Mineralogist 2000, 85, 390-395. 


\section{Graphical TOC Entry}

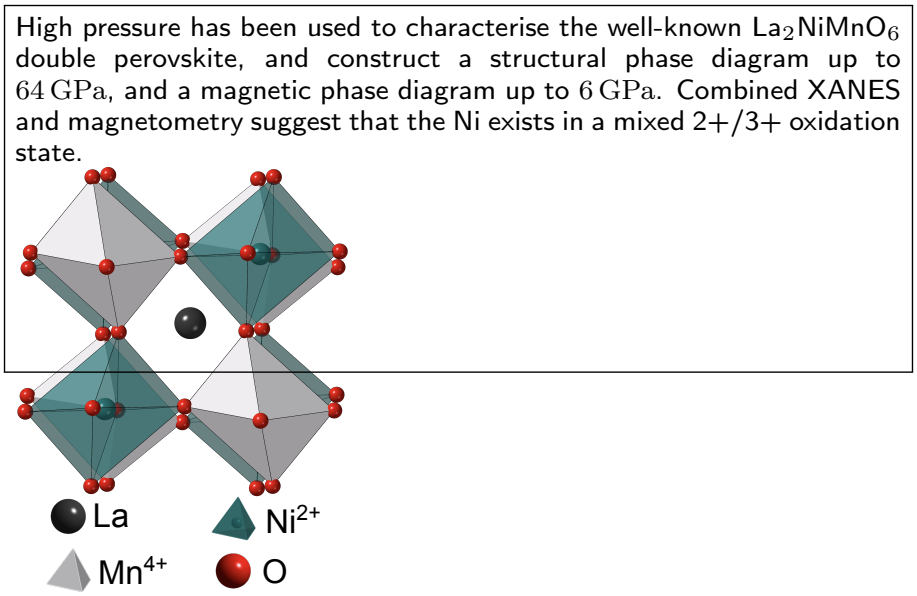




\section{High-pressure study of the elpasolite perovskite $\mathrm{La}_{2} \mathrm{NiMnO}_{6}$}

Christopher J. Ridley, ${ }^{* \dagger} \dagger$ Dominik Daisenberger, ${ }^{\ddagger}$ Craig Wilson, ${ }^{\ddagger}, \|$ Gavin B.G.

Stenning, ${ }^{\dagger}$ Gopinathan Sankar, ${ }^{\top}$ Kevin S. Knight, ${ }^{\S} \perp$ Matthew G. Tucker, ${ }^{\dagger}, \#$ Ronald I. Smith, ${ }^{\dagger}$ and Craig L. Bull ${ }^{*, \dagger}$

$\dagger$ †SIS Neutron and Muon Source, Rutherford Appleton Laboratory, Chilton, Didcot OX11 $O Q X, U K$

$\ddagger$ Diamond Light Source Ltd., Harwell Campus, Chilton, Didcot OX11 ODE, UK

9Department of Chemistry, University College London, 20 Gordon Street, WC1H OAJ London, U.K.

$\S$ Department of Earth Sciences, University College London, Gower Street, London, WC1E $6 B T, U K$

||Presently at: Atomic Weapons Establishment, Aldermaston, Berkshire RG7 4PR UK

$\perp$ Department of Earth Sciences, The Natural History Museum, Cromwell Road, London, $S W 75 B D, U K$

\#Presently at: Spallation Neutron Source, One Bethel Valley Road, MS-6475, Oak Ridge, TN 37831-6475, USA.

E-mail: christopher.ridley@stfc.ac.uk; craig.bull@stfc.ac.uk 


\section{SUPPLEMENTARY INFORMATION}

\section{Experimental details}

The sample was synthesised through hydrated nitrate decomposition using $\mathrm{La}\left(\mathrm{NO}_{3}\right) \cdot 6 \mathrm{H}_{2} \mathrm{O}(99.99 \%$ Sigma Aldrich), $\mathrm{Ni}\left(\mathrm{NO}_{3}\right) .6 \mathrm{H}_{2} \mathrm{O}$ (99.999\% Sigma Aldrich), and $\mathrm{Mn}\left(\mathrm{NO}_{3}\right) \cdot 6 \mathrm{H}_{2} \mathrm{O}$ (98\% Sigma Aldrich). The source of the NiO impurity isn't precisely known, though is most likely due to the low purity of the Mn nitrate, which was not provided with a full elemental analysis on a metals basis. Alternatively it may have formed during annealing due to the oxygen excess. A total of $4 \mathrm{~g}$ of sample was synthesised in a single batch; $90 \mathrm{mg}$ was used for the DC magnetometry measurements, a sintered pellet of $200 \mathrm{mg}$ was used for the AC magnetometry measurements, and the remainder was used for the diffraction measurements. Each loading of the Paris-Edinburgh press required approximately $500 \mathrm{mg}$.

The as synthesised sample was checked for phase purity using a Bruker D2 Phaser X-ray diffractometer using $\mathrm{Cu}$ $\mathrm{K} \alpha_{1,2}$ radiation. High temperature X-ray diffraction was performed at ambient pressure using a Rigaku Smartlab fitted with a germanium (220) monochromator, and Anton Paar HTK high-temperature stage. Low temperature neutron diffraction measurements were performed at ambient pressure using the Polaris instrument at the ISIS Neutron and Muon Source, with an AS scientific helium-flow orange-cryostat achieving temperatures in the range 4.2-290 K. The sample was loaded into a $6 \mathrm{~mm}$ diameter cylindrical vanadium can, with a RhFe sensor attached for accurate temperature control. Data were collected on warming from base temperature.

Magnetometry measurements were performed using a Quantum Design MPMS-XL7 SQUID magnetometer. Ambient pressure measurements were performed with a known mass of sample loaded into a gelatine capsule. High pressure magnetometry was performed using a turnbuckle diamond anvil cell, with a small unknown mass of sample loaded into a $\mathrm{BeCu}$ gasket between two $800 \mu \mathrm{m}$ culet diamonds. Methanol:ethanol 4:1 was used as a pressure transmitting medium, and a small ruby chip was included to determine the sample pressure [1]. To determine the relatively small magnetic contribution from the sample to the assembly, background measurements on the clean, empty cell were performed prior to loading. Through performing the same measurement routine for the empty and loaded cell, this enabled full background correction. Full details of the cell are published elsewhere [2]. Ambient pressure AC susceptibility measurements were also performed on an annealed pellet of sample using a Quantum Design PPMS-9 with ACMS module.

X-ray absorption spectroscopy measurements were performed at the Daresbury Laboratory Synchrotron Source (SRS). X-ray absorption near edge structure (XANES) spectra at the L-edge were obtained on the undulator beamline 5U.1. The samples were mixed with ultra pure graphite, pressed into discs and then mounted on aluminium holders using double sided conducting graphite tape before being placed into an ultra high vacuum chamber. The data was collected using the total electron yield method. The spectra were calibrated against an internal NiO standard with the position of the $\mathrm{L}_{I I I}$ maximum at $853.2 \mathrm{eV}$ and $\mathrm{L}_{I I}$ features at $870.3 \mathrm{eV}$ and $871.5 \mathrm{eV}$ [3].

High pressure neutron diffraction measurements were performed on both the PEARL and HRPD instruments at the ISIS Neutron and Muon Source. On the PEARL diffractometer the sample was loaded into a Ti-Zr 'null-scattering' gasket within a Paris-Edinburgh press. Deuterated methanol:ethanol 4:1 was used as a pressure medium, and a small pellet of lead was included to determine the sample pressure. Variable temperature measurements were performed using the variable-temperature insert. Temperatures above $400 \mathrm{~K}$ were measured using a high temperature gasket assembly consisting of pyrophyllite, with a graphite furnace, and the sample encapsulated in $\mathrm{MgO} . \mathrm{MgO}$ was used to determine the sample pressure, whilst the temperatures were determined through using the temperature calibrated neutron resonances of $\mathrm{Hf}$ and Ta foils included in the sample volume [4]. Full details of the press, variable temperature insert, high temperature assembly, and PEARL instrument may be found in the literature [5]. For the high pressure measurements on HRPD, a clamped piston cylinder cell was used, with $\mathrm{NaCl}$ included to determine the sample pressure, and fluorinert as a pressure transmitting medium. Full details of the cell, and it's use on HRPD with the backscattering geometry, can be found in the literature [6].

High pressure X-ray diffraction measurements were performed a the I15 beamline at the Diamond Light Source running at $30 \mathrm{keV}$. The sample was loaded into a membrane driven diamond anvil cell, with culets of $300 \mu \mathrm{m}$ diameter, into a rhenium gasket with a $100 \mu \mathrm{m}$ diameter sample hole. Neon was used as the pressure transmitting medium. A $\mathrm{CeO}_{2}$ standard was used to refine the instrument parameters. A small piece of gold was loaded into the DAC on the instrument to serve as a secondary pressure/temperature calibrant. Two sample loadings were performed, one to a maximum pressure of $40 \mathrm{GPa}$, and another to approximately $65 \mathrm{GPa}$.

High pressure Raman spectra were collected using a Princeton Instruments Acton SP-2500 spectrometer equipped with a $1800 \mathrm{~g} \mathrm{~mm}^{-1}$ grating, and a PIXIS:100B Peltier cooled CCD. The sample was excited using a 15mW narrow line width diode laser at a wavelength of $532 \mathrm{~nm}$. Two sample loadings were measured, one in $800 \mu \mathrm{m}$ and another $600 \mu \mathrm{m}$ culet diamonds used in a Merrill-Bassett cell [7]. The sample was sealed in a steel gasket, with methanol:ethanol 4:1 as a pressure medium, and a small ruby chip used to determine the sample pressure [1]. 

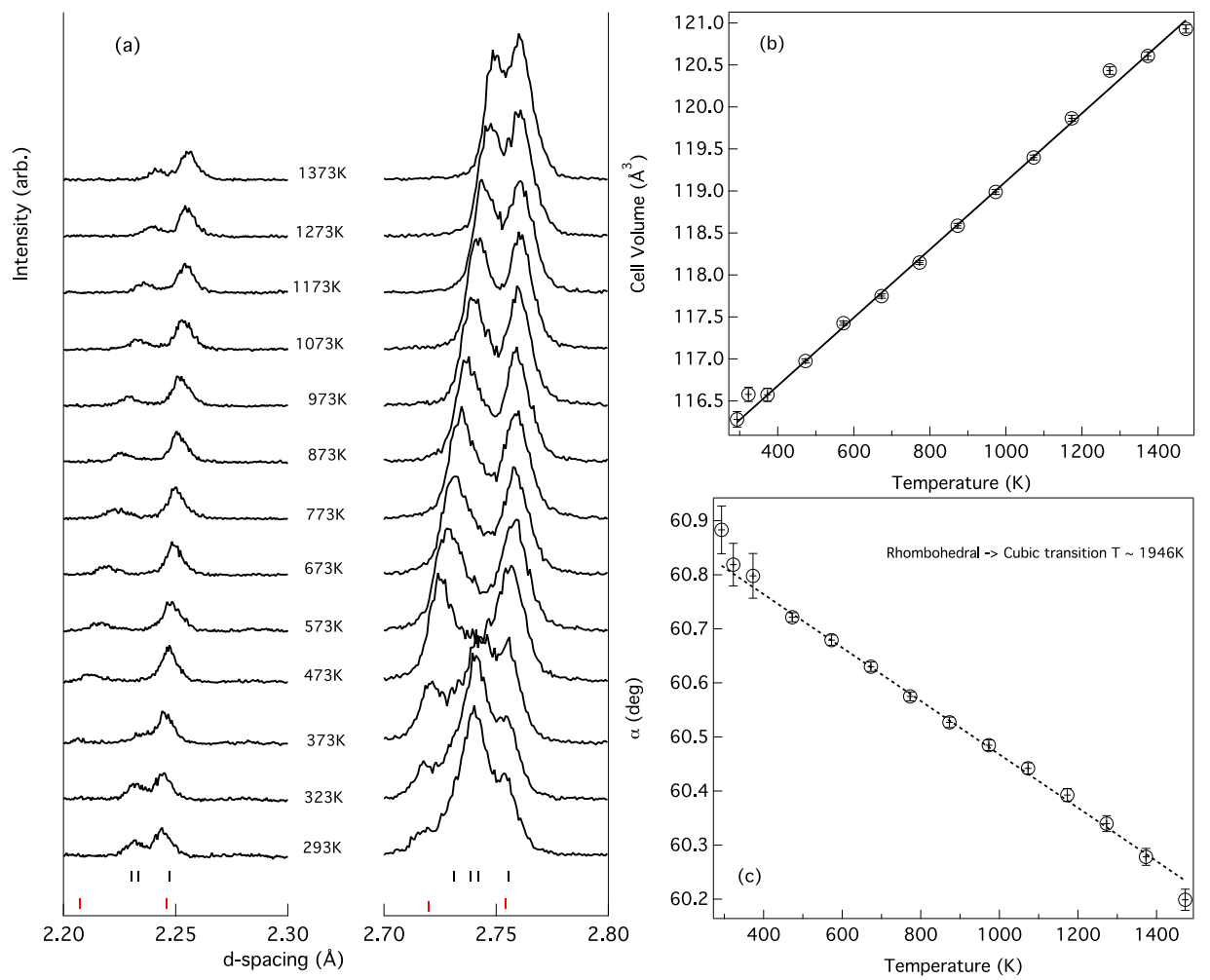

FIG. S1. (a) Representative regions of powder X-ray diffraction pattern showing the transition from mixed monoclinic/rhombohedral phase, and the subsequent shift in rhombohedral splitting towards higher symmetry as a function of temperature. Upper/lower tick marks index monoclinic/rhombohedral reflections respectively; (b) rhombohedral unit cell volume, and (c) rhombohedral angle as a function of temperature. The coloured regions represent the mixed and pure rhombohedral regions.

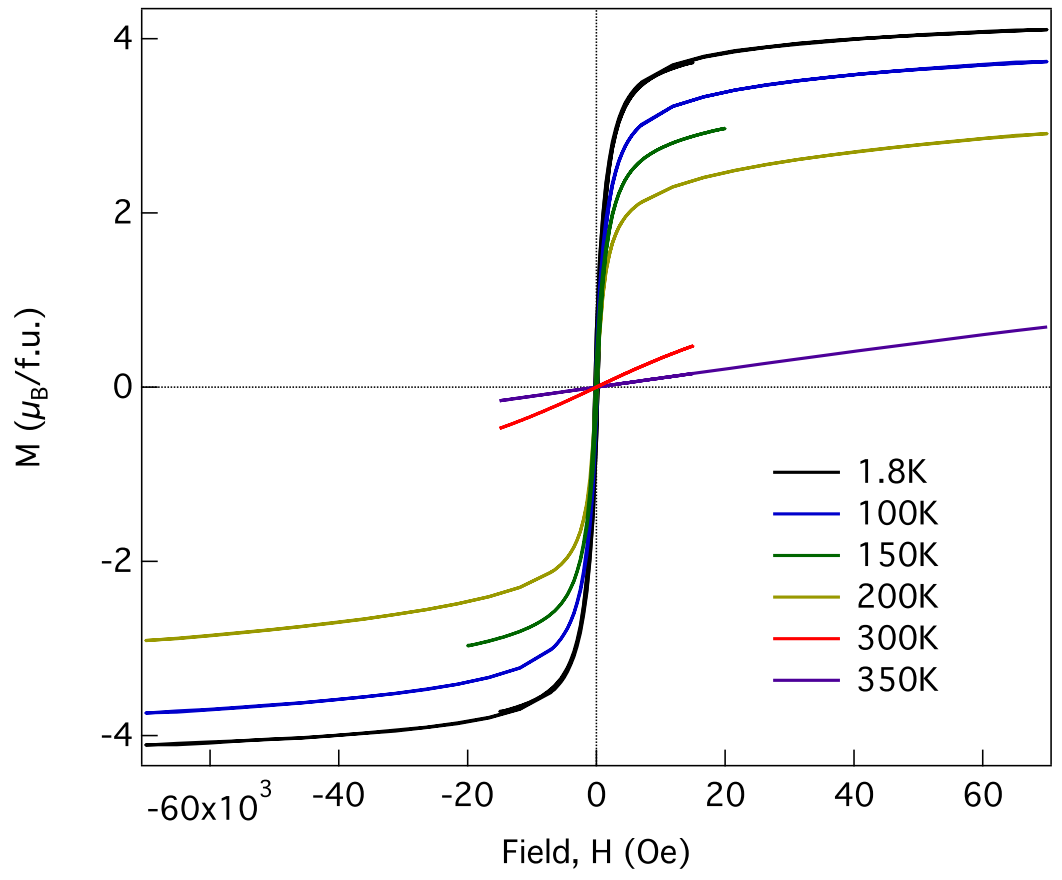

FIG. S2. Moment-field measurements at $1.8 \mathrm{~K}, 100 \mathrm{~K}, 150 \mathrm{~K}, 200 \mathrm{~K}, 300 \mathrm{~K}$, and $350 \mathrm{~K}$, measured at ambient pressure. 


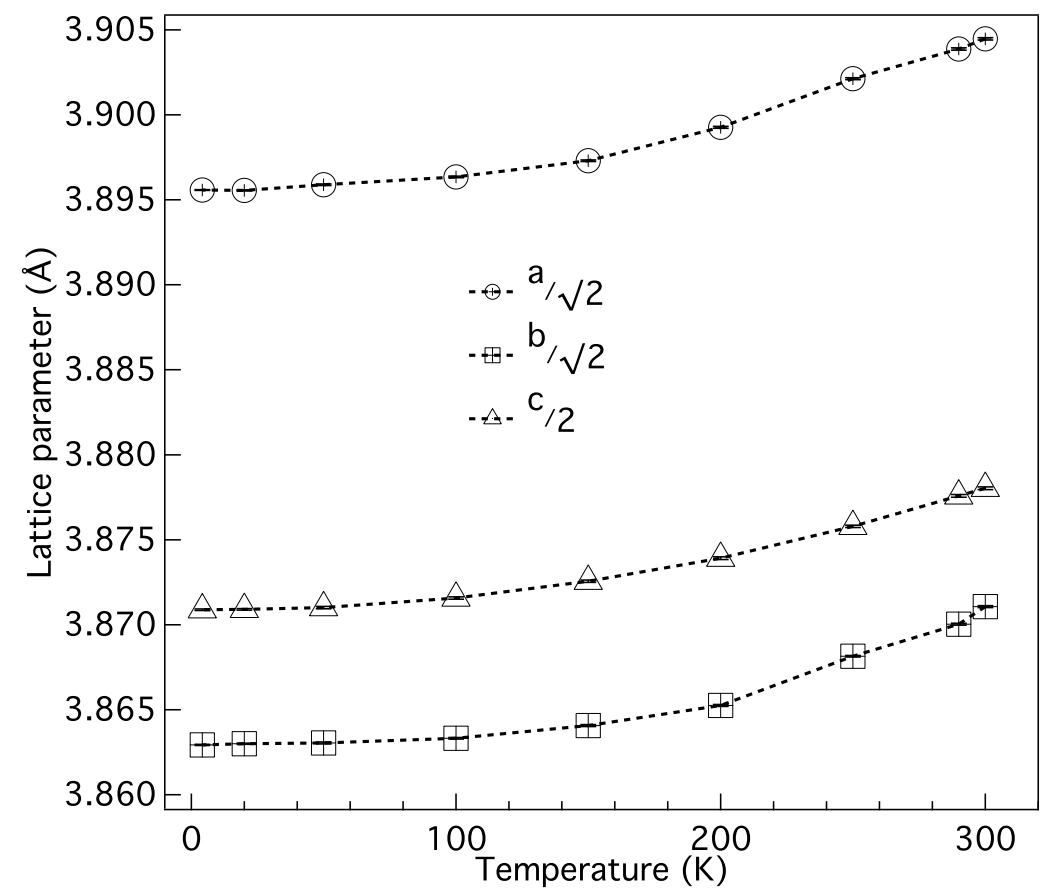

FIG. S3. Scaled lattice parameters of the monoclinic phase as function of temperature, at ambient pressure, as determined by neutron diffraction. Error bars are shown, though obscured by the symbols in some cases.

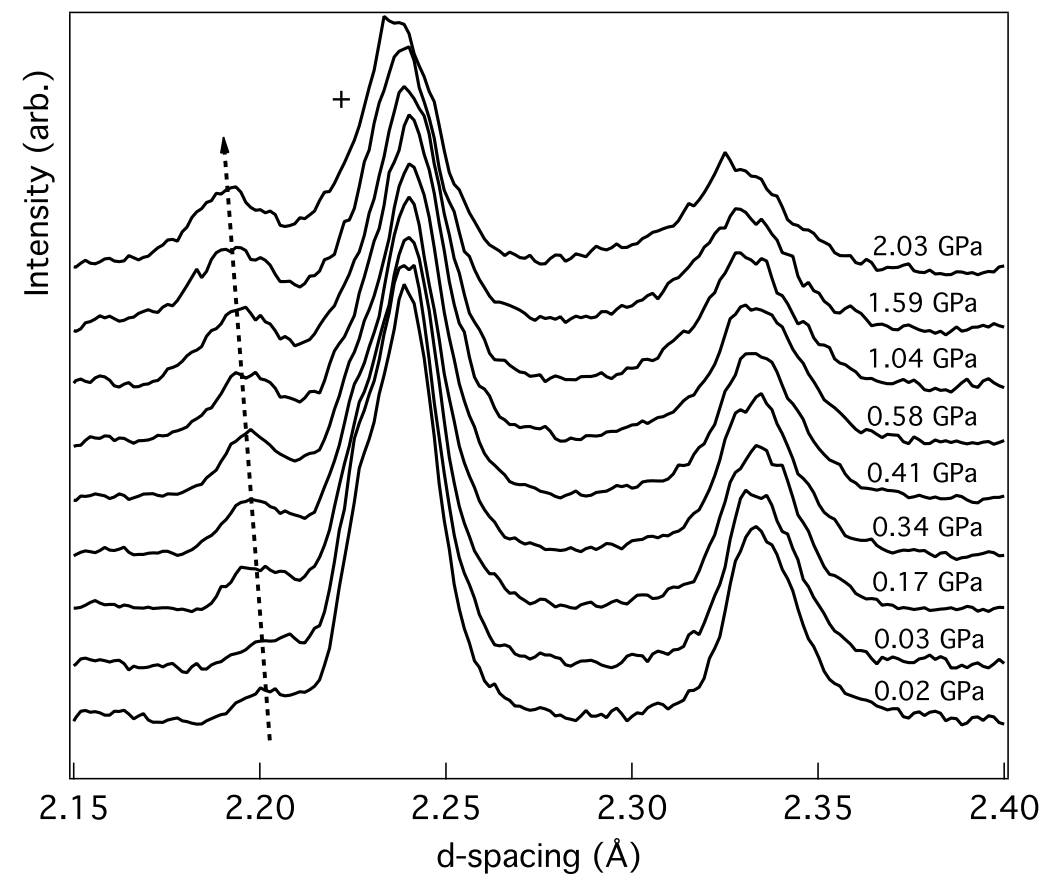

FIG. S4. Overview of ambient temperature neutron powder diffraction data over low pressure region. The dashed arrow shows the growing in of the rhombohedral phase, whilst the shoulder marked $(+)$ shows the reduction in intensity of the principal reflection of the monoclinic phase. Due to low symmetry and strong peak overlap, full Rietveld refinement was not possible over this pressure range. The two uppermost profiles are the first datasets where no monoclinic contribution was observed. 


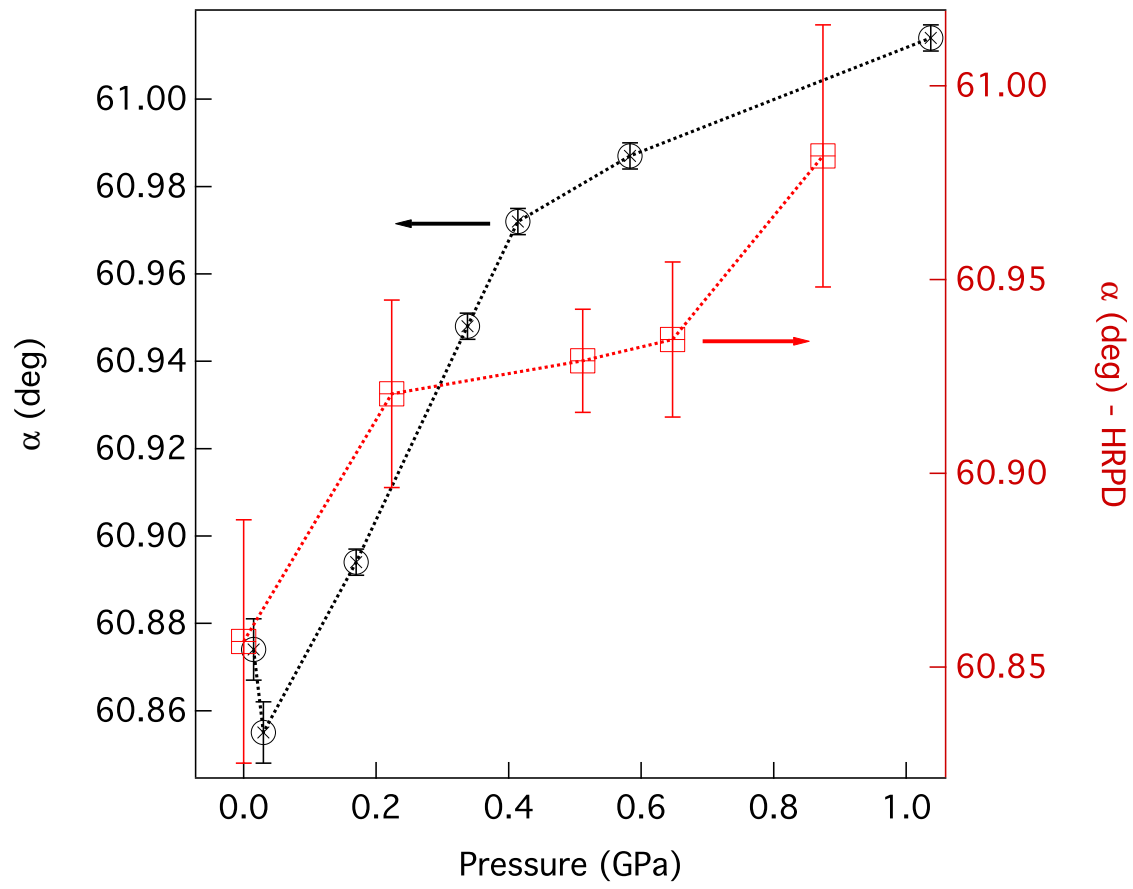

FIG. S5. LeBail extracted rhombohedral angle over mixed phase low pressure region compared with results from high resolution data [6]. The uncertainties are larger on the high resolution data due to poor statistics.

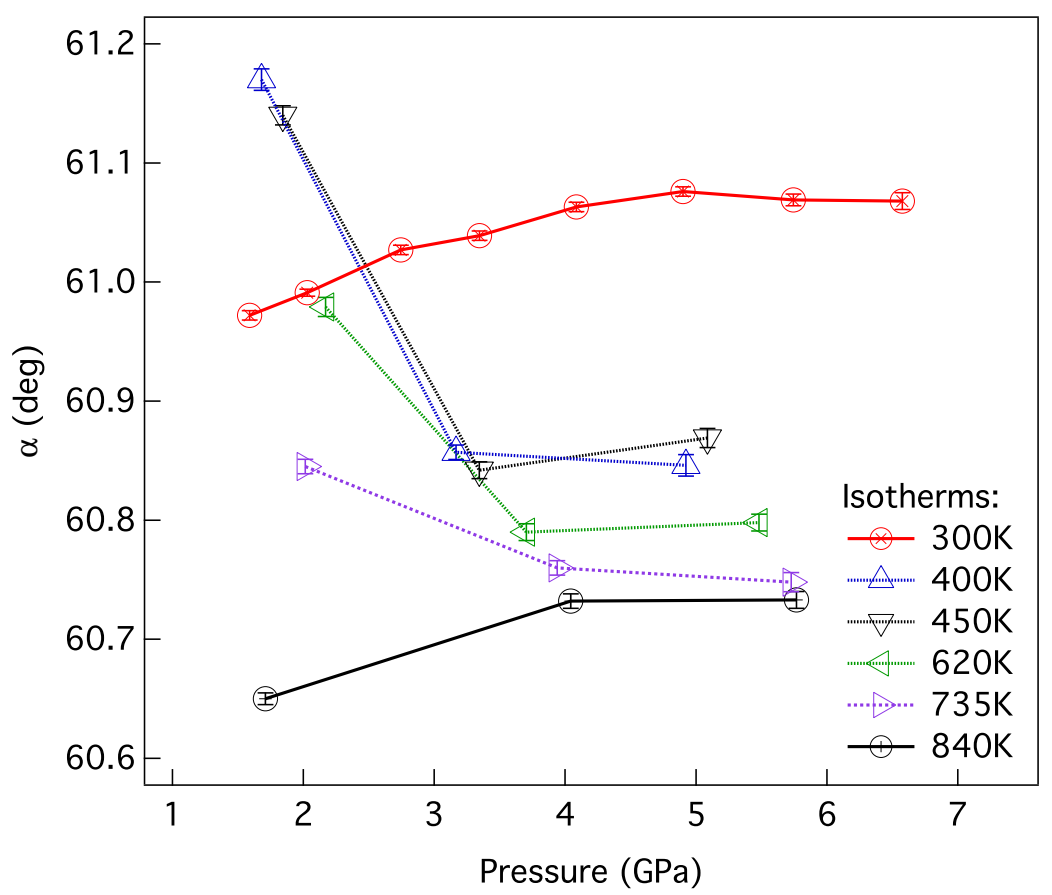

FIG. S6. Isothermal behaviour of the rhombohedral angle as a function of pressure at various temperatures between $300 \mathrm{~K}$ and $840 \mathrm{~K}$. The uppermost curve $(300 \mathrm{~K})$ was measured under highly hydrostatic conditions using methanol:ethanol as a pressure medium. The high temperature data sets were measured under only quasi-hydrostatic conditions, with $\mathrm{MgO}$ used as a pressure medium. 


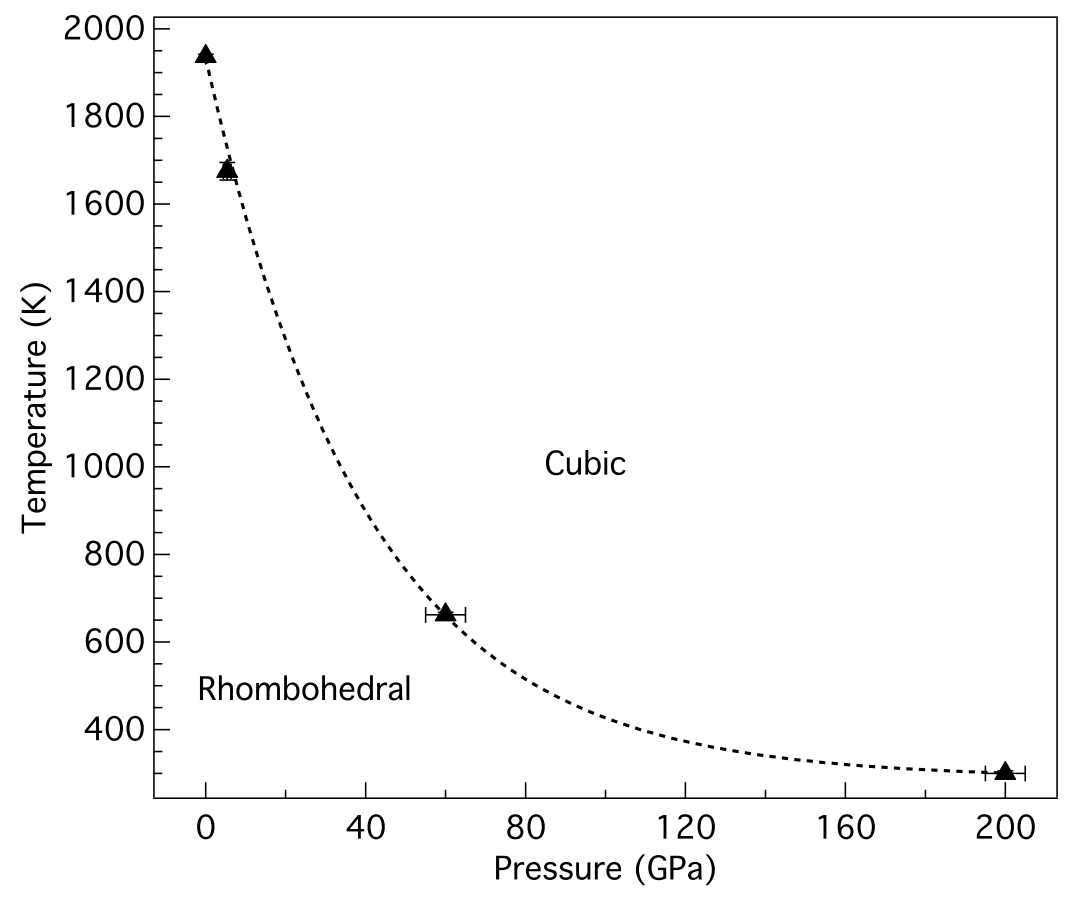

FIG. S7. Extrapolated rhombohedral to cubic transition pressure/temperature extended to very high pressures, as determined by the rhombohedral angle. The data are extrapolated from high temperature, high pressure XRD and neutron diffraction. The dashed line serves only as a guide to data.

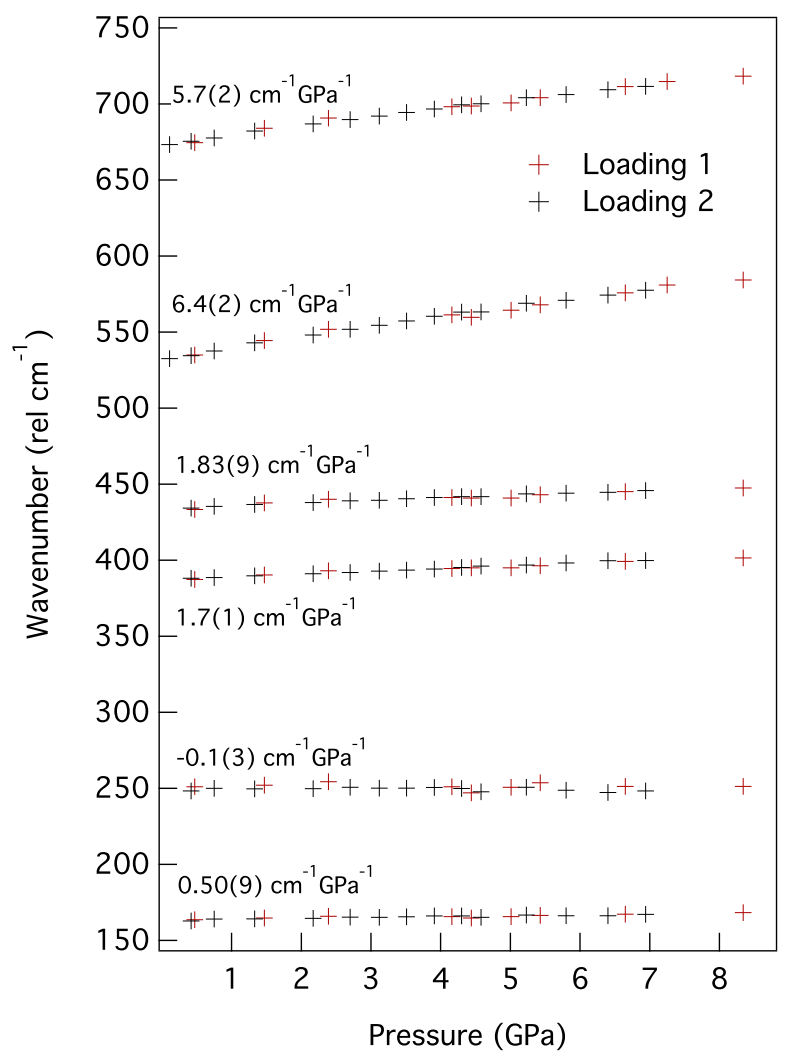

FIG. S8. Change in the Raman peak positions as a function of applied pressure. The higher energy modes are seen to shift to higher energy, whereas the weaker modes show virtually no change with pressure. While the mode at $250 \mathrm{rel} \mathrm{cm}^{-1}$ has a negative slope, within the uncertainty it too stiffens. 
TABLE S1. Rietveld refined structural parameters of the monoclinic phase of $\mathrm{La}_{2} \mathrm{NiMnO}_{6}$ as a function of temperature, determined by neutron power diffraction. The Wyckoff sites and atomic coordinates are as follows, La: $4 e(x, y, z)$, Ni1/Mn1: $2 c\left(0, \frac{1}{2}, 0\right), \mathrm{Ni} 2 / \mathrm{Mn} 2: 2 d\left(\frac{1}{2}, 0,0\right), \mathrm{O}^{1,2,3}: 4 e\left(x_{1,2,3}, y_{1,2,3}, z_{1,2,3}\right)$. The site occupancies of the transition metal sites were fixed at $85 \% \mathrm{Ni} / \mathrm{Mn}$ on $\left(0, \frac{1}{2}, 0\right) /\left(\frac{1}{2}, 0,0\right)$ respectively. The refined moments were constrained to be identical between each $\mathrm{Ni} / \mathrm{Mn}$ site, and to be only along the $b$-axis. Lattice parameters were fixed from those refined from data collected in the backscattering geometry (optimal resolution).

\begin{tabular}{c|ccccccccc}
\hline \hline $\mathrm{T}(\mathrm{K})$ & 300 & 290 & 250 & 200 & 150 & 100 & 50 & 20 & 4.2 \\
\hline$V\left(\AA^{3}\right)$ & $234.457(7)$ & $234.332(5)$ & $234.005(6)$ & $233.545(6)$ & $233.274(6)$ & $233.113(6)$ & $233.034(6)$ & $233.006(4)$ & $233.001(3)$ \\
$a(\AA)$ & $5.52175(9)$ & $5.52091(9)$ & $5.51844(8)$ & $5.51440(8)$ & $5.51161(7)$ & $5.51027(7)$ & $5.50962(7)$ & $5.50914(4)$ & $5.50918(4)$ \\
$b(\AA)$ & $5.47451(9)$ & $5.47305(8)$ & $5.47039(7)$ & $5.46629(8)$ & $5.46461(8)$ & $5.46357(7)$ & $5.46317(7)$ & $5.46311(5)$ & $5.46300(4)$ \\
$c(\AA)$ & $7.75605(16)$ & $7.75516(17)$ & $7.75158(14)$ & $7.74783(16)$ & $7.74511(14)$ & $7.74315(13)$ & $7.74202(13)$ & $7.74181(9)$ & $7.74177(8)$ \\
$\beta\left({ }^{\circ}\right)$ & $89.985(8)$ & $89.987(8)$ & $89.983(7)$ & $89.988(9)$ & $89.992(9)$ & $89.996(9)$ & $89.995(9)$ & $89.993(17)$ & $89.966(4)$ \\
$L a_{x}$ & $0.0056(6)$ & $0.0080(6)$ & $0.0079(5)$ & $-0.0096(7)$ & $-0.0084(6)$ & $-0.0081(6)$ & $-0.0069(6)$ & $-0.0066(6)$ & $-0.0053(7)$ \\
$L a_{y}$ & $0.0223(6)$ & $0.0182(7)$ & $0.0187(6)$ & $0.0225(6)$ & $0.0217(5)$ & $0.0214(5)$ & $0.0217(5)$ & $0.0219(5)$ & $0.0229(5)$ \\
$L a_{z}$ & $0.2449(6)$ & $0.2452(6)$ & $0.2442(5)$ & $0.2410(4)$ & $0.2411(4)$ & $0.2410(4)$ & $0.2414(4)$ & $0.2415(4)$ & $0.2423(5)$ \\
$O_{x}^{1}$ & $0.2791(9)$ & $0.2829(9)$ & $0.2780(8)$ & $0.259(1)$ & $0.2589(11)$ & $0.2593(11)$ & $0.2583(11)$ & $0.2582(12)$ & $0.2593(11)$ \\
$O_{y}^{1}$ & $0.2806(8)$ & $0.2858(8)$ & $0.2802(7)$ & $0.2485(8)$ & $0.2465(7)$ & $0.2469(7)$ & $0.2474(7)$ & $0.2491(7)$ & $0.2495(7)$ \\
$O_{z}^{1}$ & $0.0357(4)$ & $0.0363(5)$ & $0.0384(4)$ & $0.0384(4)$ & $0.0384(3)$ & $0.0386(3)$ & $0.0389(3)$ & $0.0388(3)$ & $0.0395(4)$ \\
$O_{x}^{2}$ & $0.2598(10)$ & $0.2558(10)$ & $0.2596(9)$ & $0.2682(11)$ & $0.2686(9)$ & $0.2693(9)$ & $0.2688(9)$ & $0.2686(9)$ & $0.2687(10)$ \\
$O_{y}^{2}$ & $0.2697(9)$ & $0.2659(9)$ & $0.2730(8)$ & $0.3113(5)$ & $0.3098(5)$ & $0.3088(4)$ & $0.3088(5)$ & $0.3082(4)$ & $0.3091(5)$ \\
$O_{z}^{2}$ & $0.4673(4)$ & $0.4656(4)$ & $0.4653(4)$ & $0.4712(5)$ & $0.4697(4)$ & $0.4692(3)$ & $0.4688(4)$ & $0.4684(3)$ & $0.4679(4)$ \\
$O_{x}^{3}$ & $0.5587(4)$ & $0.5627(4)$ & $0.5596(4)$ & $0.5443(6)$ & $0.5453(4)$ & $0.5455(4)$ & $0.5449(4)$ & $0.5456(4)$ & $0.5469(5)$ \\
$O_{y}^{3}$ & $-0.0051(12)$ & $-0.0057(12)$ & $-0.0068(11)$ & $-0.0020(11)$ & $-0.0022(10)$ & $-0.0024(10)$ & $-0.0022(10)$ & $-0.0028(10)$ & $-0.0033(9)$ \\
$O_{z}^{3}$ & $0.2524(9)$ & $0.2521(9)$ & $0.2546(8)$ & $0.2554(8)$ & $0.2558(8)$ & $0.2559(7)$ & $0.2563(8)$ & $0.2555(8)$ & $0.2549(8)$ \\
$\mu_{N i}\left(\mu_{B}\right)$ & - & - & - & $0.95(7)$ & $1.20(4)$ & $1.48(3)$ & $1.60(3)$ & $1.71(3)$ & $1.82(3)$ \\
$\mu_{M n}\left(\mu_{B}\right)$ & - & - & - & $1.08(7)$ & $1.29(4)$ & $1.58(3)$ & $1.74(3)$ & $1.86(3)$ & $1.93(4)$ \\
$R_{\mathrm{p}}(\%)$ & 3.54 & 3.01 & 3.23 & 4.33 & 4.15 & 4.23 & 4.42 & 4.38 & 4.29 \\
\hline \hline
\end{tabular}

TABLE S2. Rietveld refined structural parameters of the rhombohedral phase of $\mathrm{La}_{2} \mathrm{NiMnO}_{6}$ as a function of pressure at $300 \mathrm{~K}$, determined by neutron powder diffraction. The Wyckoff sites and atomic coordinates are as follows, La: $2 c(x, x, x)$, Ni1/Mn1: $1 a(0,0,0), \mathrm{Ni} / \mathrm{Mn} 2: 1 a\left(\frac{1}{2}, \frac{1}{2}, \frac{1}{2}\right)$, O: $6 f(x, y, z)$. The site occupancies of the transition metal sites were fixed at $85 \% \mathrm{Ni} / \mathrm{Mn}$ on $(0,0,0) /\left(\frac{1}{2}, \frac{1}{2}, \frac{1}{2}\right)$ respectively, as determined by high resolution neutron powder diffraction on the same batch of sample [8]. A full Rietveld refinement of the data below $2 \mathrm{GPa}$ was not possible due to the strong peak overlap in the mixed phase and limited instrumental resolution. The octahedral tilt around the [111] of the pseudo-cubic cell ( $\omega)$ was determined through transforming to the hexagonal setting, and then following the work of Kennedy et al. [9].

\begin{tabular}{c|cccccccc}
\hline \hline $\mathrm{P}(\mathrm{GPa})$ & $1.59(15)$ & $2.03(15)$ & $2.7(2)$ & $3.35(18)$ & $4.09(21)$ & $4.90(18)$ & $5.74(24)$ & $6.58(32)$ \\
\hline$V\left(\AA^{3}\right)$ & $115.491(15)$ & $115.224(14)$ & $114.863(16)$ & $114.472(15)$ & $114.050(17)$ & $113.549(17)$ & $113.088(19)$ & $112.562(27)$ \\
$a(\AA)$ & $5.42689(38)$ & $5.42195(36)$ & $5.41486(41)$ & $5.40824(39)$ & $5.40067(44)$ & $5.39223(44)$ & $5.38521(48)$ & $5.37687(69)$ \\
$\alpha\left(^{\circ}\right)$ & $60.972(4)$ & $60.991(3)$ & $61.027(4)$ & $61.039(4)$ & $61.063(4)$ & $61.076(4)$ & $61.069(5)$ & $61.068(7)$ \\
$L a_{x}$ & $0.24029(48)$ & $0.24128(45)$ & $0.24155(52)$ & $0.24104(47)$ & $0.24234(55)$ & $0.24302(56)$ & $0.24577(67)$ & $0.2476(11)$ \\
$O_{x}$ & $0.79691(74)$ & $0.7978(7)$ & $0.7979(8)$ & $0.7971(7)$ & $0.7969(8)$ & $0.7963(8)$ & $0.7965(8)$ & $0.7949(12)$ \\
$O_{y}$ & $0.6862(5)$ & $0.687(4)$ & $0.6879(5)$ & $0.6869(5)$ & $0.6875(5)$ & $0.6871(5)$ & $0.6892(6)$ & $0.6883(8)$ \\
$O_{z}$ & $0.2491(11)$ & $0.2539(11)$ & $0.2547(13)$ & $0.2574(12)$ & $0.2558(13)$ & $0.2605(13)$ & $0.2673(13)$ & $0.2664(21)$ \\
$\omega[111]\left(^{\circ}\right)$ & $10.696(13)$ & $10.625(17)$ & $10.544(5)$ & $10.4906(87)$ & $10.452(15)$ & $10.326(15)$ & $10.045(9)$ & $9.977(18)$ \\
$\mathrm{Ni} 1 \mathrm{Mn} 1-\mathrm{O}(\AA)$ & $2.021(4)$ & $2.0127(30)$ & $2.0069(33)$ & $2.0116(31)$ & $2.0071(34)$ & $2.0083(34)$ & $1.99084)$ & $2.003(5)$ \\
$\mathrm{Ni2} / \mathrm{Mn} 2-\mathrm{O}(\AA)$ & $1.894(4)$ & $1.8980(32)$ & $1.899(4)$ & $1.8901(34)$ & $1.889(4)$ & $1.882(4)$ & $1.887(4)$ & $1.875(6)$ \\
$\mathrm{TM} 1-\mathrm{O}-\mathrm{TM} 2\left(^{\circ}\right)$ & $161.82(14)$ & $162.01(12)$ & $162.15(14)$ & $162.12(13)$ & $162.26(15)$ & $162.28(15)$ & $162.31(18)$ & $162.49(27)$ \\
$R_{\mathrm{p}}(\%)$ & 5.54 & 5.11 & 5.54 & 5.38 & 5.69 & 5.6 & 5.79 & 7.85 \\
\hline \hline
\end{tabular}


TABLE S3. Refined lattice parameters of rhombohedral phase of $\mathrm{La}_{2} \mathrm{NiMnO}_{6}$ as a function of temperature and pressure, as measured using neutron diffraction.

\begin{tabular}{cccccccccc}
\hline \hline $\mathrm{T}(\mathrm{K})$ & $298(4)$ & $335(20)$ & $392(20)$ & $470(20)$ & $527(20)$ & $650(20)$ & $734(20)$ & $872(20)$ & $296(20)$ \\
$\mathrm{P}(\mathrm{GPa})$ & $1.4(1)$ & $1.3(4)$ & $1.7(1)$ & $1.8(1)$ & $1.9(1)$ & $2.2(1)$ & $2.0(1)$ & $1.7(1)$ & $2.8(1)$ \\
\hline$V\left(\AA^{3}\right)$ & $115.43(3)$ & $115.63(2)$ & $115.74(3)$ & $115.94(2)$ & $116.20(2)$ & $116.73(2)$ & $117.41(2)$ & $118.35(2)$ & $114.67(2)$ \\
$a(\AA)$ & $5.4185(8)$ & $5.4237(6)$ & $5.4235(8)$ & $5.4274(6)$ & $5.4339(5)$ & $5.4465(6)$ & $5.4614(5)$ & $5.4839(5)$ & $5.4161(5)$ \\
$\alpha\left(^{\circ}\right)$ & $61.161(8)$ & $61.109(8)$ & $61.157(8)$ & $61.138(8)$ & $61.076(6)$ & $60.968(8)$ & $60.857(6)$ & $60.661(5)$ & $60.918(7)$ \\
\hline $\mathrm{T}(\mathrm{K})$ & $335(20)$ & $401(20)$ & $457(20)$ & $507(20)$ & $622(20)$ & $745(20)$ & $855(20)$ & $298(4)$ & $348(20)$ \\
$\mathrm{P}(\mathrm{GPa})$ & $3.0(1)$ & $3.2(1)$ & $3.3(1)$ & $3.4(1)$ & $3.7(1)$ & $3.9(1)$ & $4.0(1)$ & $4.6(1)$ & $4.8(1)$ \\
\hline$V\left(\AA^{3}\right)$ & $114.67(2)$ & $114.86(2)$ & $114.99(3)$ & $115.12(2)$ & $115.44(3)$ & $115.64(2)$ & $116.096(14)$ & $113.40(2)$ & $113.44(2)$ \\
$a(\AA)$ & $5.4184(5)$ & $5.4212(6)$ & $5.4244(7)$ & $5.4279(5)$ & $5.4332(7)$ & $5.4380(4)$ & $5.4459(4)$ & $5.3953(5)$ & $5.3972(6)$ \\
$\alpha\left({ }^{\circ}\right)$ & $60.864(6)$ & $60.863(6)$ & $60.838(6)$ & $60.801(5)$ & $60.793(6)$ & $60.752(5)$ & $60.731(5)$ & $60.937(7)$ & $60.906(7)$ \\
\hline $\mathrm{T}(\mathrm{K})$ & $397(20)$ & $448(20)$ & $497(20)$ & $615(20)$ & $730(20)$ & $840(20)$ & $934(20)$ & $1059(20)$ & $1177(20)$ \\
$\mathrm{P}(\mathrm{GPa})$ & $4.9(1)$ & $5.1(1)$ & $5.2(1)$ & $5.5(1)$ & $5.7(1)$ & $5.8(1)$ & $5.4(1)$ & $5.4(1)$ & $5.4(1)$ \\
\hline$V\left(\AA^{3}\right)$ & $113.56(2)$ & $113.64(2)$ & $113.73(2)$ & $114.04(2)$ & $114.41(2)$ & $114.69(2)$ & $115.33(2)$ & $115.95(2)$ & $116.59(2)$ \\
$a(\AA)$ & $5.4014(6)$ & $5.4016(5)$ & $5.4037(5)$ & $5.4108(5)$ & $5.4191(5)$ & $5.4236(5)$ & $5.4365(6)$ & $5.4521(4)$ & $5.4654(5)$ \\
$\alpha\left({ }^{\circ}\right)$ & $60.848(8)$ & $60.873(7)$ & $60.858(6)$ & $60.802(6)$ & $60.741(6)$ & $60.737(6)$ & $60.671(6)$ & $60.523(4)$ & $60.442(4)$ \\
\hline \hline
\end{tabular}

TABLE S4. Refined lattice parameters of rhombohedral phase of $\mathrm{La}_{2} \mathrm{NiMnO}_{6}$ as a function of pressure, as measured using $\mathrm{X}$-ray diffraction.

Loading 1

\begin{tabular}{|c|c|c|c|c|c|c|c|c|c|c|c|}
\hline $\mathrm{P}(\mathrm{GPa})$ & $2.17(8)$ & \multicolumn{2}{|l|}{$2.57(7)$} & \multicolumn{2}{|c|}{$2.87(9)$} & \multicolumn{2}{|l|}{$3.3(1)$} & $3.8(1)$ & $4.4(2)$ & $5.3(5)$ & $12.3(9)$ \\
\hline$V\left(\AA^{3}\right)$ & $115.069(11)$ & ) $115.068(1$ & 11) & 114.700 & (7) & 114.481 & $(7)$ & $114.216(6)$ & $113.767(5)$ & $113.202(5)$ & $109.390(6)$ \\
\hline$a(\AA)$ & $5.4289(3)$ & $5.4288(3$ & & 5.4213( & & 5.4174( & & $5.4130(2)$ & $5.4057(2)$ & $5.3966(2)$ & $5.3352(2)$ \\
\hline$\alpha\left(^{\circ}\right)$ & $60.757(3)$ & $60.757(3$ & & 60.799 & & 60.810( & & $60.817(2)$ & $60.822(2)$ & $60.826(2)$ & $60.828(2)$ \\
\hline $\mathrm{P}(\mathrm{GPa})$ & $16.5(6)$ & $22.3(7)$ & & $5.2(7)$ & & $0.3(4)$ & & $4.6(5)$ & $39.6(5)$ & & \\
\hline$V\left(\AA^{3}\right)$ & $107.665(6) 1$ & $105.688(6)$ & 104 & $1.592(6)$ & 103 & $3.205(7)$ & 101. & $.879(5) \quad 10$ & $00.462(5)$ & & \\
\hline$a(\AA)$ & $5.3065(2)$ & $5.2733(2)$ & 5.2 & $549(2)$ & & $2313(2)$ & 5.20 & $2094(2) \quad 5.1$ & 18531(14) & & \\
\hline$\alpha\left(^{\circ}\right)$ & $60.843(2)$ & $60.854(2)$ & 60. & $.857(2)$ & & $.865(2)$ & 60. & $849(2)$ & $50.844(2)$ & & \\
\hline
\end{tabular}

Loading 2

\begin{tabular}{|c|c|c|c|c|c|c|c|c|c|}
\hline $\mathrm{P}(\mathrm{GPa})$ & $5.4(6)$ & $9.6(5)$ & $14.7(5)$ & $20.7(9)$ & $25.4(3$ & & $30(1)$ & $35.0(3)$ & $39.7(3)$ \\
\hline$V\left(\AA^{3}\right)$ & $113.029(14)$ & $111.130(7)$ & $108.830(9)$ & $106.616(15)$ & 5) 104.890 & $9(13)$ & $103.072(14)$ & $101.98(2)$ & $100.889(5)$ \\
\hline$a(\AA)$ & $5.3926(3)$ & $5.3609(2)$ & $5.3233(2)$ & $5.2866(4)$ & 5.2573 & $3(4)$ & $5.2256(4)$ & $5.2084(5)$ & $5.1899(2)$ \\
\hline$\alpha\left(^{\circ}\right)$ & $60.858(2)$ & $60.888(2)$ & $60.900(3)$ & $60.909(5)$ & 60.926 & $5(4)$ & $60.955(4)$ & $60.92(6)$ & $60.917(2)$ \\
\hline $\mathrm{P}(\mathrm{GPa})$ & $44.7(2)$ & $49.3(4)$ & $54.3(4)$ & $59.4(2)$ & $64.0(5)$ & \multicolumn{2}{|c|}{$67.4(3)$} & & \\
\hline$V\left(\AA^{3}\right)$ & $99.609(4)$ & $98.703(2)$ & $97.228(6)$ & $96.156(4) 9$ & $95.088(4)$ & \multicolumn{2}{|c|}{$94.740(4)$} & & \\
\hline$a(\AA)$ & $5.16793(12)$ & $5.15359(14)$ & $5.1286(2)$ & $5.1099(2) 5$ & $5.0924(3)$ & \multicolumn{2}{|c|}{$5.0872(2)$} & & \\
\hline$\alpha\left(^{\circ}\right)$ & $60.915(2)$ & $60.878(3)$ & $60.858(3)$ & $60.852(3) \quad 6$ & $60.812(6)$ & \multicolumn{2}{|c|}{$60.783(6)$} & & \\
\hline
\end{tabular}

TABLE S5. Refined lattice parameters from rhombohedral phase at high temperatures at approximately 60 GPa as determined by XRD.

\begin{tabular}{ccc}
\hline \hline $\mathrm{P}(\mathrm{GPa})$ & $60.0(5$ & $60.0(5)$ \\
$\mathrm{T}(\mathrm{K})$ & $600(6)$ & $720(6)$ \\
\hline$V\left(\AA^{3}\right)$ & $95.299(11)$ & $95.781(11)$ \\
$a(\AA)$ & $5.10696(14)$ & $5.1222(2)$ \\
$\alpha\left(^{\circ}\right)$ & $60.524(3)$ & $60.351(7)$ \\
\hline
\end{tabular}


TABLE S6. Refined lattice parameters of rhombohedral phase of $\mathrm{La}_{2} \mathrm{NiMnO}_{6}$ as a function of temperature, as measured using X-ray diffraction.

\begin{tabular}{ccccccccc}
\hline \hline $\mathrm{T}(\mathrm{K})$ & 293 & 323 & 373 & 473 & 573 & 673 & 773 & 873 \\
\hline$V\left(\AA^{3}\right)$ & $116.28(9)$ & $116.58(8)$ & $116.57(8)$ & $116.98(2)$ & $117.43(3)$ & $117.75(3)$ & $118.15(2)$ & $118.59(3)$ \\
$a(\AA)$ & $5.44(3)$ & $5.45(4)$ & $5.45(3)$ & $5.460(6)$ & $5.469(7)$ & $5.476(6)$ & $5.484(6)$ & $5.493(7)$ \\
$\alpha\left(^{\circ}\right)$ & $60.88(4)$ & $60.82(4)$ & $60.80(4)$ & $60.721(8)$ & $60.679(9)$ & $60.629(9)$ & $60.575(8)$ & $60.527(9)$ \\
\hline $\mathrm{T}(\mathrm{K})$ & 973 & 1073 & 1173 & 1273 & 1373 & 1473 \\
\hline$V\left(\AA^{3}\right)$ & $118.99(3)$ & $119.39(3)$ & $119.87(4)$ & $120.43(5)$ & $120.61(5)$ & $120.93(5)$ \\
$a(\AA)$ & $5.501(7)$ & $5.509(7)$ & $5.518(8)$ & $5.529(10)$ & $5.534(11)$ & $5.54(14)$ \\
$\alpha\left(^{\circ}\right)$ & $60.485(9)$ & $60.442(10)$ & $60.392(12)$ & $60.339(15)$ & $60.28(2)$ & $60.19(2)$ \\
\hline \hline
\end{tabular}


TABLE S7. Rietveld refined structural parameters of the rhombohedral phase of $\mathrm{La}_{2} \mathrm{NiMnO}_{6}$ as a function of pressure and temperature, determined by neutron powder diffraction. The Wyckoff sites and atomic coordinates are as follows, La: $2 c$ $(x, x, x), \mathrm{Ni} 1 / \mathrm{Mn} 1: 1 a(0,0,0), \mathrm{Ni} / \mathrm{Mn} 2: 1 a\left(\frac{1}{2}, \frac{1}{2}, \frac{1}{2}\right), \mathrm{O}: 6 f(x, y, z)$. The site occupancies of the transition metal sites were fixed at $85 \% \mathrm{Ni} / \mathrm{Mn}$ on $(0,0,0) /\left(\frac{1}{2}, \frac{1}{2}, \frac{1}{2}\right)$ respectively, as determined by high resolution neutron powder diffraction on the same batch of sample [8]. The octahedral tilt around the [111] of the pseudo-cubic cell $(\omega)$ was determined through transforming to the hexagonal setting, and then following the work of Kennedy et al. [9].

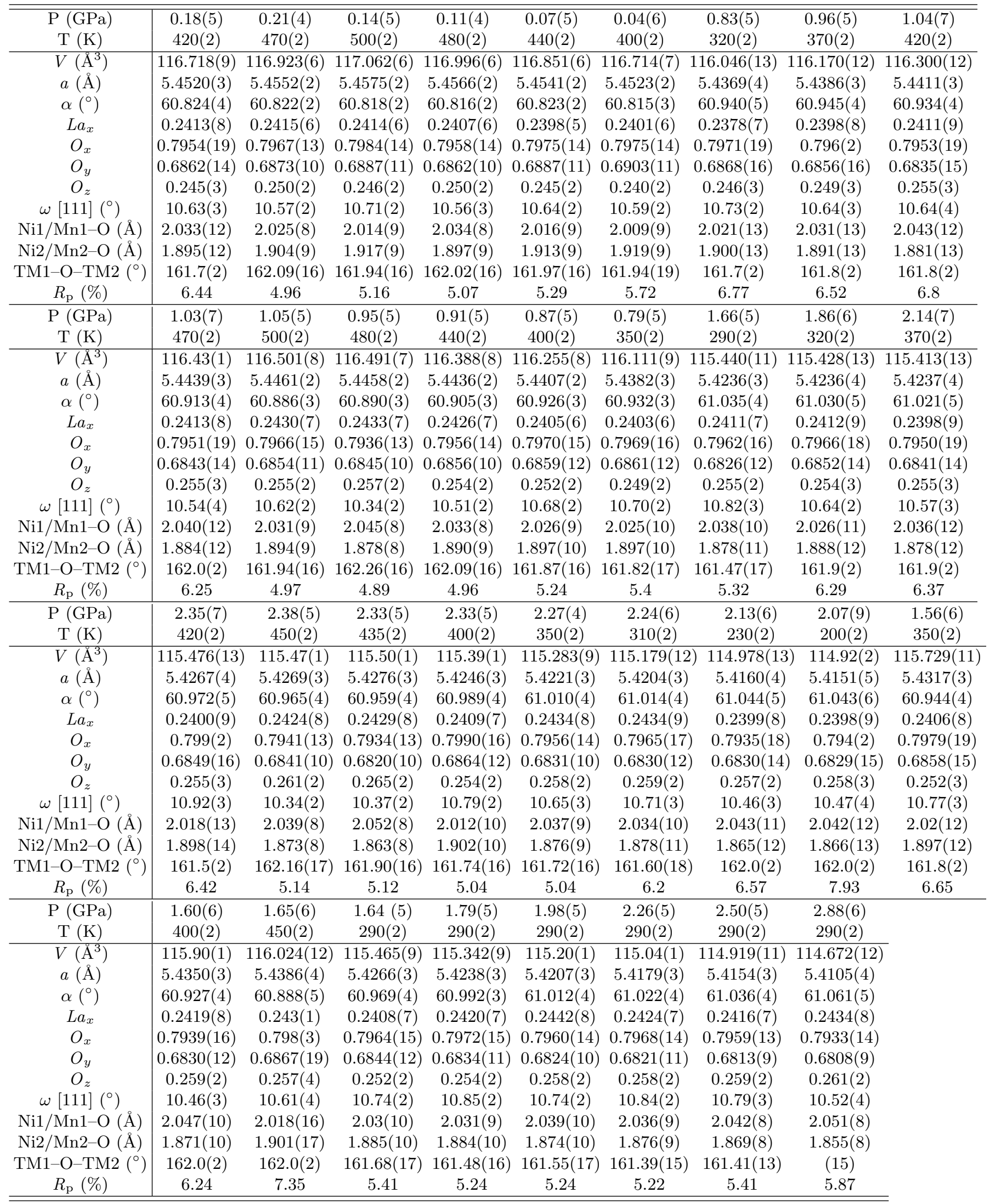




\section{REFERENCES}

[1] G.J. Piermarini, S. Block, J.D. Barnett, and R.A. Forman, "Calibration of the pressure dependence of the R1 ruby fluorescence line to 195 kbar," Journal of Applied Physics 46, 2774-2780 (1975).

[2] G. Giriat, W. Wang, J.P. Attfield, A.D. Huxley, and K.V. Kamenev, "Turnbuckle diamond anvil cell for high-pressure measurements in a superconducting quantum interference device magnetometer," Review of Scientific Instruments 81, 073905 (2010).

[3] G. Van der Laan, J. Zaanen, G.A. Sawatzky, R. Karnatak, and J-M. Esteva, "Comparison of X-ray absorption with x-ray photoemission of nickel dihalides and NiO," Physical Review B 33, 4253 (1986).

[4] Y. Le Godec, M.T. Dove, D.J. Francis, S.C. Kohn, W.G. Marshall, A.R. Pawley, G.D. Price, S.A.T. Redfern, N. Rhodes, N.L. Ross, P.F. Schofield, E. Schooneveld, G. Syfosse, M.G. Tucker, and M.D. Welch, "Neutron diffraction at simultaneous high temperatures and pressures, with measurement of temperature by neutron radiography," Mineralogical Magazine 65, 737-748 (2001).

[5] C.L. Bull, N.P. Funnell, M.G. Tucker, S. Hull, D.J. Francis, and W.G. Marshall, "PEARL: the high pressure neutron powder diffractometer at ISIS," High Pressure Research 36, 493-511 (2016).

[6] C.L. Bull, A.D. Fortes, C.J. Ridley, I.G. Wood, D.P. Dobson, N.P. Funnell, A.S. Gibbs, C.M. Goodway, R. Sadykov, and K.S. Knight, "High-resolution neutron-diffraction measurements to 8 kbar," High Pressure Research 37, 486-494 (2017).

[7] L. Merrill and W.A. Bassett, "Miniature diamond anvil pressure cell for single crystal X-ray diffraction studies," Review of Scientific Instruments 45, 290-294 (1974).

[8] C.L. Bull, D. Gleeson, and K.S. Knight, "Determination of B-site ordering and structural transformations in the mixed transition metal perovskites $\mathrm{La}_{2} \mathrm{CoMnO}_{6}$ and $\mathrm{La}_{2} \mathrm{NiMnO}_{6}$," Journal of Physics: Condensed Matter 15, 4927 (2003).

[9] B.J. Kennedy, C.J. Howard, K.S. Knight, Z. Zhang, and Q. Zhou, "Structures and phase transitions in the ordered double perovskites $\mathrm{Ba}_{2} \mathrm{Bi}^{I I I} \mathrm{Bi}^{V} \mathrm{O}_{6}$ and $\mathrm{Ba}_{2} \mathrm{Bi}^{I I I} \mathrm{Sb}^{V} \mathrm{O}_{6}$," Acta Crystallographica Section B 62, 537-546 (2006). 